\title{
AN INVESTIGATION OF POLY(N-ISOPROPYLACRYLAMIDE) FOR APPLICATIONS WITH MICROFLUIDIC PAPER-BASED ANALYTICAL DEVICES
}

\author{
A Thesis \\ presented to \\ the Faculty of California Polytechnic State University, \\ San Luis Obispo \\ In Partial Fulfillment \\ of the Requirements for the Degree \\ Masters of Science in Polymers and Coatings Science
}

by

Haydn T. Mitchell

June 2014 
(C)2014

Haydn T. Mitchell

ALL RIGHTS RESERVED 
COMMITTEE MEMBERSHIP

TITLE:

An Investigation of Poly( $\mathrm{N}$-isopropylacylamide)

for Applications with Microfluidic Paper-based

Analytical Devices

AUTHOR: Haydn T. Mitchell

DATE SUBMITTED: June $17^{\text {th }}, 2014$

COMMITTEE CHAIR: Andres W. Martinez, PhD

Assistant Professor, Department of Chemistry

and Biochemistry

MEMBER:

Philip J. Costanzo, PhD

Associate Professor, Department of Chemistry

and Biochemistry

MEMBER:

Raymond H. Fernando, PhD

Professor, Endowed Chair of Polymers

and Coatings Program 


\title{
ABSTRACT
}

An Investigation of Poly(N-isopropylacrylamide) for Applications with

\author{
Microfluidic Paper-based Analytical Devices
}

Haydn T. Mitchell

$N, N^{\prime}$-methylenebisacrylamide-crosslinked poly(N-isopropylacrylamide), also known as P(NIPAM), was developed as a fluid delivery system for use with microfluidic paper-based analytical devices (microPADs). MicroPADs are postage-stamp-sized devices made out of paper that can be used as platforms for low-cost, simple-to-use point-of-care diagnostic assays. $\mathrm{P}(\mathrm{NIPAM})$ is a thermally responsive polymer that absorbs aqueous solutions at room temperature and will expel the solutions to microPADs when heated. The fluid delivery characteristics of P(NIPAM) were assessed, and P(NIPAM) was able to deliver multiple solutions to microPADs in specific sequences or simultaneously in a laminar-flow configuration. P(NIPAM) was then shown to be suitable for delivering four classes of reagents to microPADs: small molecules, enzymes, antibodies and DNA. P(NIPAM) successfully delivered a series of standard concentrations of glucose $(0-5 \mathrm{mM})$ to microPADs equipped to perform a colorimetric glucose assay. The results of these tests were used to produce an external calibration curve, which in turn was used to determine accurately the concentrations of glucose in sample solutions. P(NIPAM) successfully delivered fluorescein-labeled IgG and fluorescein-labeled oligonucleotides (20 base pairs) to microPADs in a variety of concentrations. P(NIPAM) also successfully delivered horseradish peroxidase (HRP) to microPADs, and it was determined that HRP could be stored in P(NIPAM) for 35 days 
with minimal loss in activity. The combination of P(NIPAM) with microPADs will allow for more complex assays to be performed with minimal user input, will facilitate the preparation of external calibration curves in the field, and may be useful in extending the shelf life of microPADs by stabilizing reagents. 


\section{ACKNOWLEDGMENTS}

The completion of my education at Cal Poly is the ending of a great time in my life. I was at Cal Poly for six years and I have many people to thank for such a wonderful experience. I think it is appropriate to start with my first friend and mentor, my mother. I want to thank you for your love, patience, and hard work. My time in SLO would have not been possible without your assistance. You have given me such a strong start in life.

To my grandparents, I would like to thank you for your guidance throughout school and your support in all my endeavors.

To Madison and David, you are both my brothers. Looking back on our experience here at Cal Poly I couldn't imagine having been anywhere else or with anyone else. I'm excited to see what we all do next.

To Cory, I have learned a lot from you in these last three years, both as a scientist and as a person. I know for sure I would not have accomplished what I have without you by my side. Thank you for keeping me centered when I needed it most.

To Dr. Martinez, thank you for this graduate experience. You gave me room to imagine. You may not have known it, but you helped cultivate my inquisitive spirit, a major factor in my success. Lastly, you always listened to me, and then worked through a problem with me, not for me. For that I am grateful. 
To Dr. Costanzo, thank you for pushing the quality of my work. I respect and appreciate your style of teaching. Your instruction was by far one of the most influential I have had at Cal Poly. 


\section{TABLE OF CONTENTS}

Pages

LIST OF TABLES $\ldots \ldots \ldots \ldots \ldots \ldots \ldots \ldots \ldots \ldots \ldots \ldots \ldots \ldots \ldots \ldots \ldots \ldots \ldots$

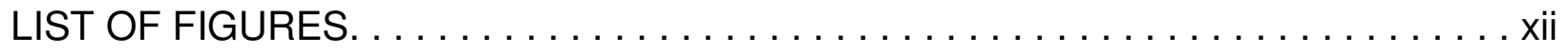

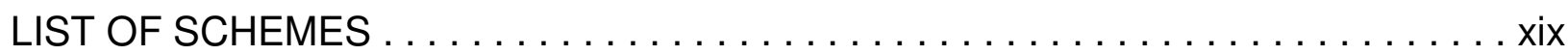

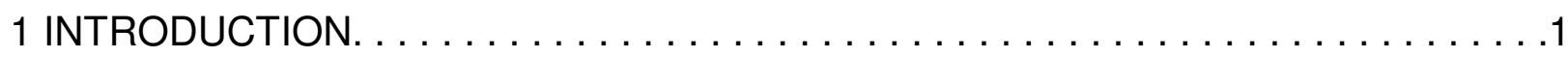

1.1 Microfluidic Paper-based Analytical Devices $\ldots \ldots \ldots \ldots \ldots \ldots \ldots 1$

1.1.1 Microfluidics. . . . . $\ldots \ldots \ldots \ldots \ldots \ldots \ldots \ldots \ldots \ldots \ldots$

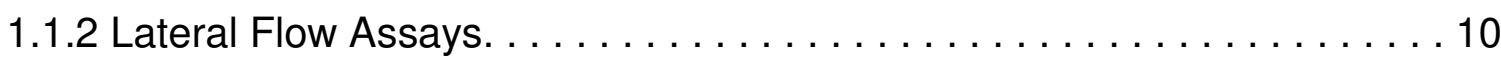

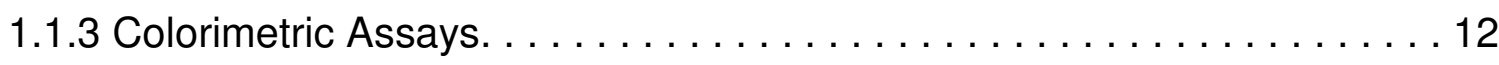

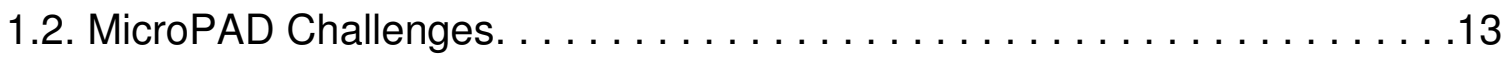

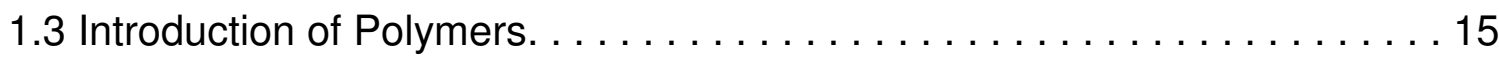

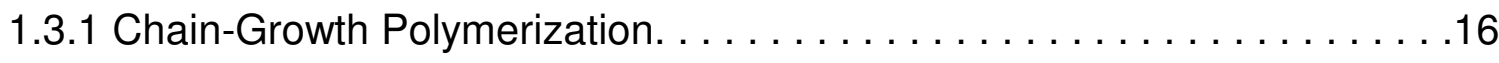

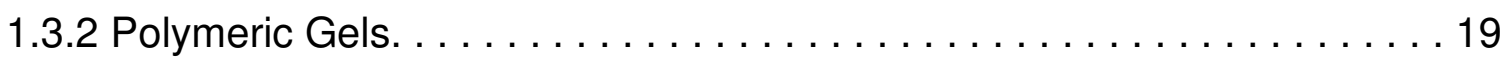

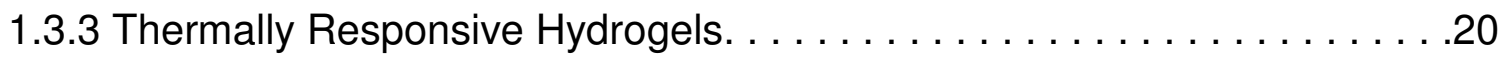

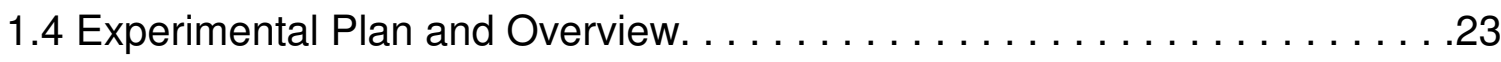

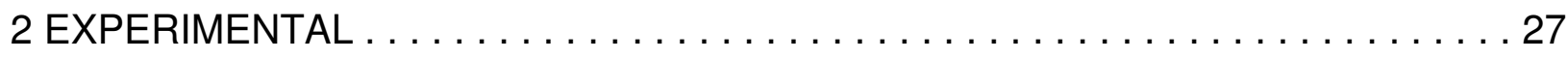

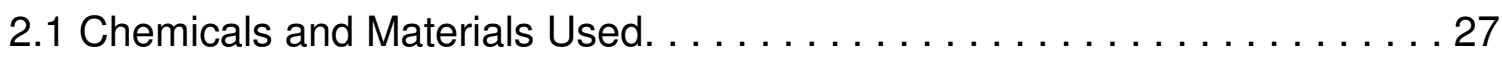




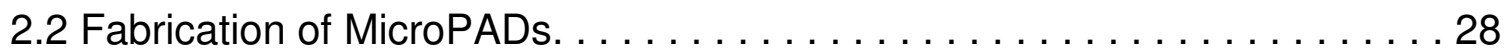

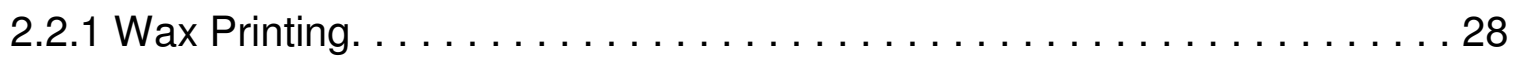

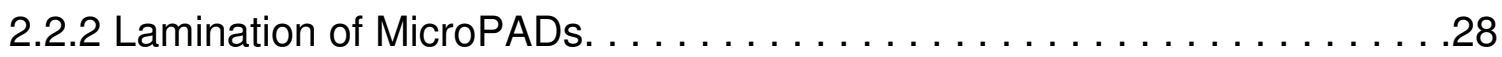

2.3 ImageJ Quantitative Analysis. . . . . . . . . . . . . . . . . . . 29

2.4 Synthesis and Handling of Poly(N-isopropylacrylamide) . . . . . . . 30

2.4.1 Synthesis of Poly(N-isopropylacrylamide) $\ldots \ldots \ldots \ldots \ldots \ldots \ldots \ldots$

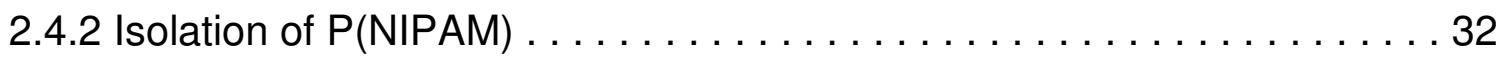

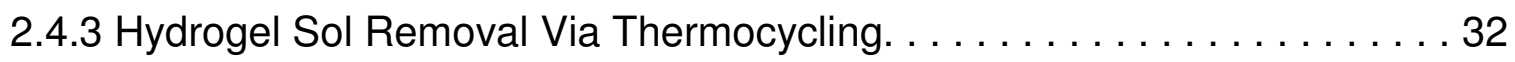

2.4.4 Characterization of P(NIPAM) Fluid Delivery $\ldots \ldots \ldots \ldots \ldots \ldots \ldots 33$

2.4.5 Loading Reagents into $\mathrm{P}(\mathrm{NIPAM}) \ldots \ldots \ldots \ldots \ldots \ldots \ldots \ldots \ldots \ldots$

2.5 Application of P(NIPAM) to MicroPADs $\ldots \ldots \ldots \ldots \ldots \ldots \ldots \ldots \ldots$

2.5.1 Preliminary Fluid Delivery Assessment of P(NIPAM) $\ldots \ldots \ldots \ldots 35$

2.5.2 Assessment of P(NIPAM) as a Delivery System

for Clinically Relevant Assays $\ldots \ldots \ldots \ldots \ldots \ldots \ldots \ldots \ldots \ldots \ldots \ldots \ldots \ldots \ldots \ldots$

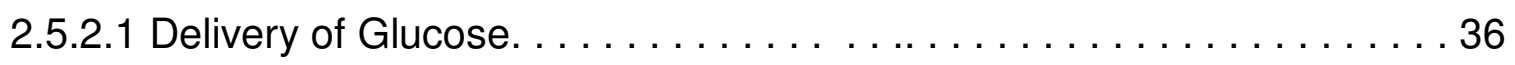

2.5.2.2 Delivery of Fluorescein-labeled $\lg G \ldots \ldots \ldots \ldots \ldots \ldots$ 
2.5.2.3 Delivery of Fluorescein-labeled DNA . . . . . . . . . . . . . 39

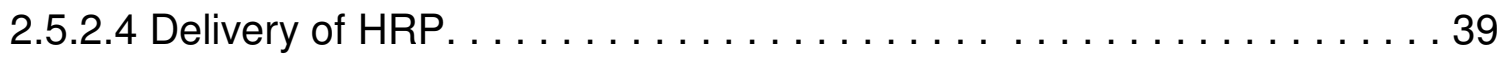

2.5.3 Assessment of P(NIPAM) as a Storage System for HRP. . . . . . .41

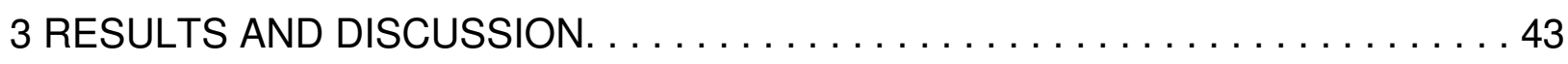

3.1 Poly(N-isopropylacrylaimide) Synthesis,

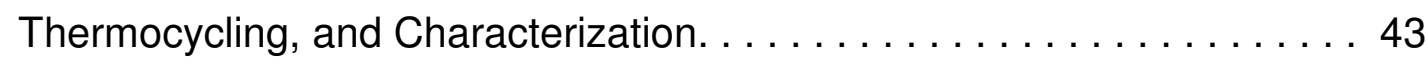

3.2 Preliminary P(NIPAM) Applications with MicroPADs. . . . . . . . . 52

3.3 Delivery of Clinically Relevant Molecules using P(NIPAM) . . . . . . .57

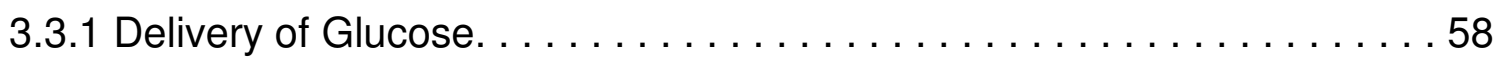

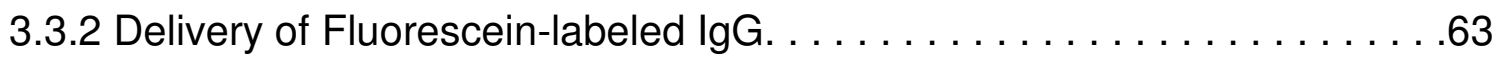

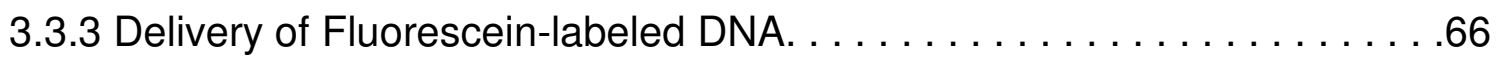

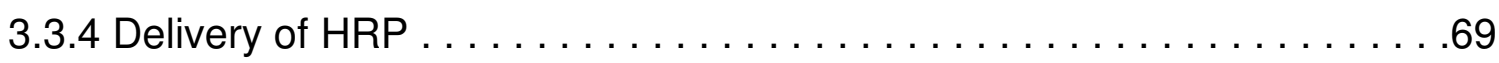

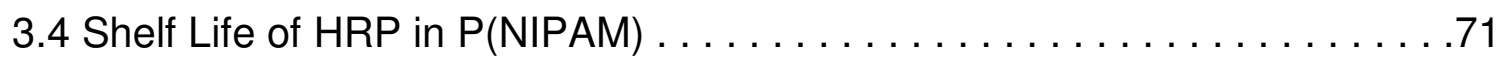

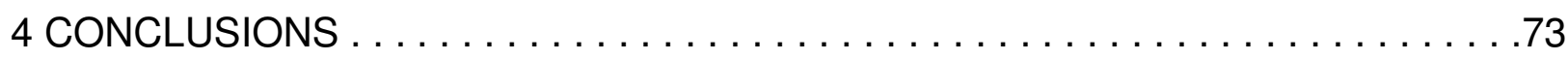

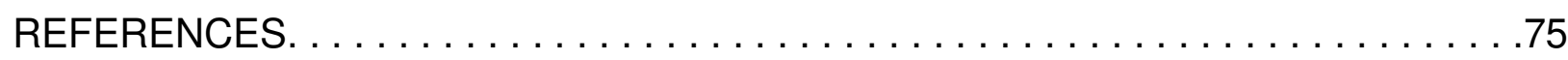




\section{LIST OF TABLES}

Tables

Page

1.1.1.1. A collection of qualitative ratios that when solved for a given system elucidates the dominating physical forces at play. ${ }^{16}$ Reprinted with permission from Review of Modern Physics, 2005, 77, 977-1026. Copyright 2005 American Physical Society.

3.3.1.1. The calculation of the concentration of glucose in samples using the $\mathrm{P}$ (NIPAM) produced glucose calibration curve. Results represent the mean of 3 measurements.

3.3.2.1. The determined concentrations of antibody samples based on their fluorescence intensity signal using calibration curves produced using P(NIPAM)-delivered and a pipette-delivered standard antibody solutions. Results represent the mean of 3 measurements.

3.3.3.1. Determined concentrations of DNA samples based on their fluorescence intensity signal using the calibration curves produced from P(NIPAM)-delivered and a pipette-delivered DNA. Results represent the mean of 3 measurements. 


\section{LIST OF FIGURES}

Figures

1.1.1. A postage stamp-sized microPAD after conducting duplicate colorimetric assays for glucose (left) and protein (right) from a single sample. ${ }^{2}$ Reprinted with permission from Analytical Chemistry, 2008, 80, 3699-3707. Copyright 2008 American Chemical Society.

1.1.1.1. (a) This schematic illustrates the use of laminar flow and diffusivity to separate particles. ${ }^{16,20,21}$ Reprinted with permission from Review of Modern Physics, 2005, 77, 977-1026. Copyright 2005 American Physical Society. (b) This device mixes fluids using wavy channels to form a gradient. ${ }^{22}$ Reprinted with permission from Analytical Chemistry, 2000, 15, 8311-8316. Copyright 2000 American Chemical Society.

1.1.2.1. Depicted is the layout and process of a gold nanoparticle lateralflow assay showing a generic interaction between an analyte, antibodies, and antigen. ${ }^{23}$ Reprinted with permission from Eline P. Meulenberg, Chapter 11, Antibodies Applications and New Developments, Bentham E-Books.

1.2.1. (a) A microfluidic device on a lab bench top using a syringe pump drive liquid through the device. (b) An end-user delivering a liquid sample to a microPAD using a micropipette. 
1.3.1.1. Depiction of the various polymer topologies. ${ }^{33}$ Reprinted with permission from George Odian, Principles of Polymerization, John Wiley \& Sons, Inc. ${ }^{34}$

1.3.3.1. Plot of temperature versus the volume fraction of polymer in solution. UCST and LCST graphs shown for clarification of the reversible switch of solubility.

1.3.3.2. An increase in isopropyl rotation is caused by thermal excitation. The fast rotating isopropyl group disturbs the water within the hydrogel matrix and causes the chains to collapse.

1.4.1. The dotted line indicates the path of dissection for a discrete sample. The P(NIPAM) sample shown has a $11.03 \mathrm{~mm}$ diameter.

3.1.1. $\quad$ P(NIPAM) samples molded via straws to illustrate the array of P(NIPAM) diameters available, and the scale of the gels after synthesis and processing. (a) Three reaction vials containing the three P(NIPAM) diameters of $11.03 \mathrm{~mm}, 7.24 \mathrm{~mm}$, and 5.35 mm respectively, stored in $\mathrm{DI} \mathrm{H}_{2} \mathrm{O}$. (b) An unsheathed P(NIPAM) sample with a diameter of $11.03 \mathrm{~mm}$ followed by sheathed samples of P(NIPAM) with diameters of $11.03 \mathrm{~mm}$, $7.24 \mathrm{~mm}$, and $5.35 \mathrm{~mm}$ respectively. 
3.1.2. Plot of the mass percent expelled versus the number of thermocycles for all three diameters of P(NIPAM). Data points represent the mean of 3 measurements and error bars represent one standard deviation from the mean.

3.1.3. Plot of the initial mass of a $P($ NIAPM) sample versus the average mass of fluid expelled. The mass of fluid delivered increases with an increase in the mass of the sample. Diminishing returns are observed. Data points represent the mean of 3 measurements. Data was fit with logarithmic trend lines to aid the eye.

3.1.4. Plot of the initial mass of P(NIPAM) sample versus the average percent mass delivered. The average percent mass delivered was relative to the initial mass of the sample. The mass of fluid delivered relative to the initial mass of the P(NIPAM) sample precipitously decreases and levels near $0.5 \mathrm{~g}$ for each of the three diameters. Data points represent the mean of 3 measurements and error bars represent one standard deviation from the mean. Data was fit with power function trend lines to aid the eye. 
3.1.5. Plot of heating time versus average mass delivered from 5.35 $\mathrm{mm}$ diameter samples. Each data point represents a single measurement. Data was fit with logarithmic trend lines to aid the eye.

3.1.6. Plot of heating time versus average mass delivered from 7.24 $\mathrm{mm}$ diameter samples. Each data point represents a single measurement. Data was fit with logarithmic trend lines to aid the eye.

3.1.7. Plot of heating time versus average mass delivered from 11.03 $\mathrm{mm}$ diameter samples. Each data point represents a single measurement. Data was fit with logarithmic trend lines to aid the eye.

3.2.1. A sequence of images documenting the diminishing wicking rate of fluid delivered to a circular sample addition zone of a microPAD by $\mathrm{P}(\mathrm{NIPAM})$ at $40{ }^{\circ} \mathrm{C}$. The diminishing wicking rate is typical of fluids in microPADs.

3.2.2. A plot of time versus distance wicked for fluids delivered to the same device by three different methods. Data points for the pipette-delivered solution $(\diamond)$ represent the mean of 3 trials. Data points for both P(NIPAM)-delivered solutions represent a single measurement. The pipette-delivered solution was borrowed from Camplisson et al. ${ }^{39}$ 
3.2.3. A sequence of images documenting three loaded P(NIPAM) samples delivering fluids simultaneously. These fluids then participate in laminar flow. Dye diffusion can be seen across the channel between the three fluids.

3.2.4. A sequence of images documenting three loaded P(NIPAM) samples delivering fluids simultaneously. These fluids then participate in plug-reagent delivery. Dye diffusion is minimal between the solutions.

3.3.1.1. Scanned image of the P(NIPAM) delivered glucose solutions onto a paper-based 96-well plate (section 2.5.2.1). Concentration of glucose delivered increases from row $\mathrm{A}$ to $\mathrm{F}$. Condition 1, Condition 2 and Condition 3 was applied to columns $1-3,4-6$, and 7-9, respectively. Reaction zone A1 was contaminated by a $1 \mathrm{mM}$ glucose loaded P(NIPAM) sample.

3.3.1.2. Plot of color intensity versus the glucose concentration for Conditions 1-3. Data points represent the mean of 3 measurements and error bars represent one standard deviation from the mean. 
3.3.1.3. Plot of concentration of glucose versus color intensity for P(NIPAM)-delivered solutions. The equation of the best-fit line for this plot may be used to determine the concentration of glucose in samples for concentrations between $0 \mathrm{mM}$ and $5 \mathrm{mM}$ glucose. Data points represent the mean of 3 measurements and error bars represent one standard deviation from the mean.

3.3.2.1. Plot of fluorescence intensity versus concentration for P(NIPAM)-delivered fluorescein-labeled antibody $(\diamond)$ and pipette-delivered fluorescein-labeled antibody (匹). Data points represent the mean of 3 measurements and the error bars represent one standard deviation from the mean.

3.3.3.1. Plot of fluorescence intensity versus concentration for P(NIPAM)-delivered fluorescein-labeled DNA $(\diamond)$ and pipettedelivered DNA ( $\mathbf{\square})$. Data points represent the mean of 3 measurements and error bars represent one standard deviation from the mean.

3.3.4.1. Image of P(NIPAM)-delivered HRP (section 2.5.2.2) in the wells of a paper-based 96-well plate. Column 10 is the External Solution trials. Column 11 is the Solution Storage trials. Column 12 is the Nonsolution Storage trails. 
3.3.4.2. Plot of color intensity versus the number of trials a sample was used. Data points represent the mean of 3 measurements and error bars represent one standard deviation from the mean.

3.4.1. Plot of color intensity versus time for three conditions (Nonsolution Storage $(\bullet)$, Solution Storage $(\boldsymbol{\square})$, and External Solution $(\bullet))$. Data points represent the mean of 3 measurements and error bars represent one standard deviation from the mean. 


\section{LIST OF SCHEMES}

$\begin{array}{lll}\text { Schemes } & \text { Page }\end{array}$

2.4.1.1. Synthetic plan for poly(N-isopropylacrylamide). 31 


\section{INTRODUCTION}

The populations of many developing nations are currently experiencing public healthcare hardships. ${ }^{1}$ Millions of people in developing nations die every year from preventable and treatable diseases. ${ }^{1-3}$ The first step toward treating a disease effectively is proper diagnosis, but many of the common diagnostic tests used in developed nations, such as the United States, are not appropriate for use in developing nations due to the cost of the tests, and the lack of infrastructure and trained personnel. ${ }^{1-3}$ In an attempt to address this problem, researchers have developed an inexpensive platform for diagnostic tests called microfluidic paperbased analytical devices (microPADs). ${ }^{2-4}$ The work presented in this thesis adds new capabilities to microPADs by introducing the use of a thermally-responsive hydrogel, poly(N-isopropylacrylamide) or P(NIPAM), as a system for storing and then delivering fluids to microPADs. P(NIPAM)'s fluid delivery capabilities, ability to deliver clinically relevant analytes to microPADs, and ability to store analytes for extended periods of time before delivering them to microPADs were all explored and characterized.

\subsection{Microfluidic Paper-based Analytical Devices}

MicroPADs are a new class of microfluidic devices that are made out of paper, patterned into hydrophilic channels and test zones bounded by hydrophobic barriers (Figure 1.1.1). ${ }^{4}$ The channels wick fluids by capillary action so a microPAD can take a small volume of a sample and combine it with a set of 
reagents in order to detect an analyte. MicroPADs require only small volumes of sample, they can conduct multiple assays simultaneously, they are inexpensive, and they are easy to use. ${ }^{2,4}$ These features are found independently in three established technologies: i) microfluidic devices (Section 1.2), ii) lateral-flow assays (e.g. pregnancy test) (Section 1.3) and iii) dipstick colorimetric assays (e.g. pH litmus paper) (Section 1.4), but microPADs conveniently combine all these features into a single device, while providing a few unique advantages of their own. These advantages include: i) scalable fabrication using existing paperprocessing and printing technologies ${ }^{3}$, ii) low cost (Whatman No. 1 chromatography paper costs $\$ 8.20 / \mathrm{m}^{2}$ from Sigma Aldrich), iii) compatibility with many chemical and biochemical assays,,$^{2,4-10}$ and iv) easy disposal via incineration, a great characteristic for medical sanitation standards. ${ }^{2}$ All of these features make microPADs an attractive platform for simple diagnostic tests with much promise for commercialization. The large-scale production of microPADs could answer the need for low-cost diagnostics in the developing world.

MicroPADs typically contain a sample inlet, for introducing the sample to the device; a network of channels, for distributing and processing the sample; and test zones, for storing reagents and providing an area where chemical reactions may take place in order to identify analytes. To run an assay, a liquid sample (e.g. blood, urine, saliva or sweat) can be added to the sample inlet, or the sample inlet can be dipped into a drop of sample. The sample is then wicked into the device without the need for pumps or batteries and automatically 
combines with the reagents present on the device. Specific color changes are then generated chemically on the device in response to analytes present in the sample, and the results are displayed in the test zones. From these results, a diagnosis could be made and proper medical treatment could be administered. A variant of this scenario is a type of telemedicine (section 1.7.2) where the results could instead be imaged with a smartphone at the point-of-care (POC) and the image could be sent to an off-site clinic for interpretation.

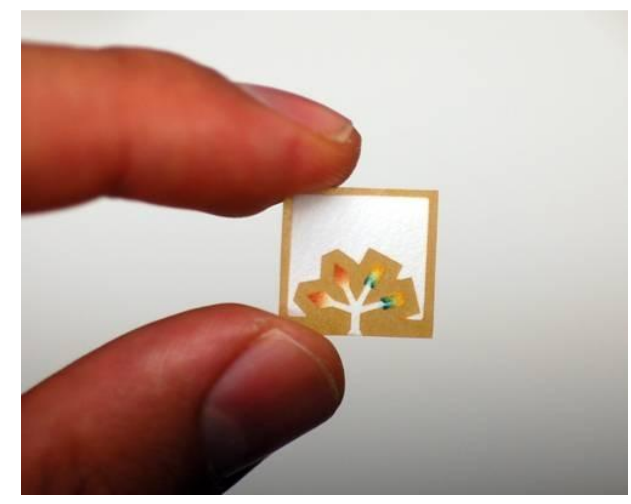

Figure 1.1.1 A postage stamp-sized microPAD after conducting duplicate colorimetric assays for glucose (left) and protein (right) from a single sample. ${ }^{2}$ Reprinted with permission from Analytical Chemistry, 2008, 80, 3699-3707. Copyright 2008 American Chemical Society.

To fabricate microPADs, paper, which is inherently hydrophilic, must be patterned with a hydrophobic material to confine and control the wicking of aqueous solutions. A variety of methods for patterning paper have been 
established, these include: (1) photolithography, (2) plotting, (3) ink jet etching(4) plasma treatment, (5) paper cutting, (6) wax printing, (7) ink jet printing, (8) flexographic printing, (9) screen printing, and (10) laser treatment. ${ }^{2,4,11-15}$ All of these methods have their advantages and disadvantages. Only wax printing will be discussed further as it was the only method of patterning used throughout this body of work.

Wax printing is the process of printing a wax on the surface of paper, and then heating the paper to melt and reflow the wax so that it seeps into the matrix of the paper and coats the cellulose fibers, thus creating a hydrophobic barrier. ${ }^{13}$ Wax printing is a rapid, efficient and inexpensive method, which is advantageous because prototypes may be fabricated rapidly and then adjusted according to the device requirements. Additionally, this process is scalable to produce large quantities of microPADs in a roll-to-roll process.

The name microfluidic paper-based analytical devices are addressed to clarify how these devices are microfluidic. MicroPAD's features are larger than classic microfluidic devices with channels commonly being $2 \mathrm{~mm}$ in width and several millimeters in length. This would not qualify as microfluidic. The term does have relevance to the nature of the devices though considering the devices commonly require milliliters of fluid to operate and have pores within the cellulosic medium that are on the micro- and nanometer scale. Furthermore, the devices due operate mostly according to the physical laws of microfluidics. Overall though, the term microfluidic was used to make the name of the technology more attractive. 
Traditional microfluidic devices, lateral-flow assays and dipstick colorimetric assays, inspired the development of microPADs so these technologies are reviewed in more detail below to provide additional context for the discussion of microPADs.

\subsubsection{Microfluidics}

Microfluidics is a field that involves manipulating small volumes (i.e. microto picoliters) of fluid in micro- and nanometer-sized channels. ${ }^{16,17}$ Microfluidic devices owe their origin in part to microanalytical methods such as gel permeation chromatography (GPC), high-performance liquid chromatography (HPLC), and gas chromatography (GC) which employ miniaturized plumbing to leverage the advantages of small scale analysis. ${ }^{17}$ The more recent progenitors of microfluidics include molecular analysis, biodefense, molecular biology, and microelectronics. ${ }^{17}$ These devices draw and touch upon numerous fields, from analytical chemistry, biochemistry, and biology to materials science, engineering, and fluid physics. Incredible feats have been accomplished with microfluidic devices: polymerase chain reaction on the nanoliter scale, cell separations, and even organic synthesis. ${ }^{16,17}$ Microfluidic devices possess numerous desirable capabilities, which stem from their small size. For example, experiments can be performed with small amounts of sample and reagent, the devices create little waste and have minimal space requirements as well as low cost, expedient execution, and high sensitivity. ${ }^{16}$ With microfluidic devices it is also easy to perform multiple tasks simultaneously on one chip. Another interesting property 
of microfluidic devices is the unique fluid manipulation abilities and physical fluid phenomena that occur at the small scale. The small fluid volumes and micrometer feature dimensions give raise to surface chemistry effects controlling fluid and particle action. ${ }^{16}$ The physics of small volumes have been the center of much study with the advent of microfluidics and nanofluidics. ${ }^{16-19} \mathrm{~A}$ discussion of the microfluidic physics is out of the scope of this thesis, but to give an idea of the breadth of physical phenomena that are relevant to fluid motion in microfluidic devices, a large set of dimensionless numbers are given in Table 1.1.1.1 The dimensionless numbers have been developed to simplify the assessment of ruling physical phenomena within devices allowing for function to be controlled through design more easily and readily. 
Table 1.1.1.1. A collection of qualitative ratios that when solved for a given system elucidates the dominating physical forces at play. ${ }^{16}$ Reprinted with permission from Review of Modern Physics, 2005, 77, 977-1026. Copyright 2005 American Physical Society.

\begin{tabular}{ccc}
\hline \hline Re & Reynolds & inertial/viscous \\
$\mathrm{Pe}$ & Péclet & convection/diffusion \\
$\mathrm{Ca}$ & capillary & viscous/interfacial \\
$\mathrm{Wi}$ & Weissenberg & polymer relaxation time/shear rate time \\
$\mathrm{De}$ & Deborah & polymer relaxation time/flow time \\
$\mathrm{El}$ & elasticity & elastic effects/inertial effects \\
$\mathrm{Gr}$ & Grashof & Re for buoyant flow \\
$\mathrm{Ra}$ & Rayleigh & Pe for buoyant flow \\
$\mathrm{Kn}$ & Knudsen & slip length/macroscopic length \\
\hline \hline
\end{tabular}

Three ratios of particular importance to this work is the Capillary, Reynolds, and Péclet numbers - a ratio of the viscous to the interfacial forces, the inertial forces to the viscous forces, and the convection to the diffusion forces of a fluid, respectively. ${ }^{16}$ For microPADs the Capillary number is less than one, meaning the interfacial forces dominate the viscous forces. So in this porous medium there is capillary driven distribution of fluids with low speeds. The 
Reynolds number is also far less than one indicating viscous interactions overcome the inertial component of the fluid. With such a small inertial component there is no convection. This lack of convection results in the Péclet number being less than one and diffusion being the major driving force for mixing. Diffusion is a time dependent process and many microfluidic devices have minimal sample diffusion due to the speed at which fluids pass through their miniscule plumbing. ${ }^{16}$ Since the wicking rate for microPADs is slow, any distance traveled takes an appreciable amount of time so diffusion occurs to a larger extent than in conventional microfluidic devices. Such a situation, with a Capillary, Reynolds, and Péclet number that is less than one results in the emergence of ideal flow, otherwise known as laminar flow. Laminar flow is the absence of any turbulence or eddies within the flow stream. ${ }^{16}$ Figure 1.1.1.1 gives examples of how laminar flow may be used or overcome in microfluidics. 
(a)

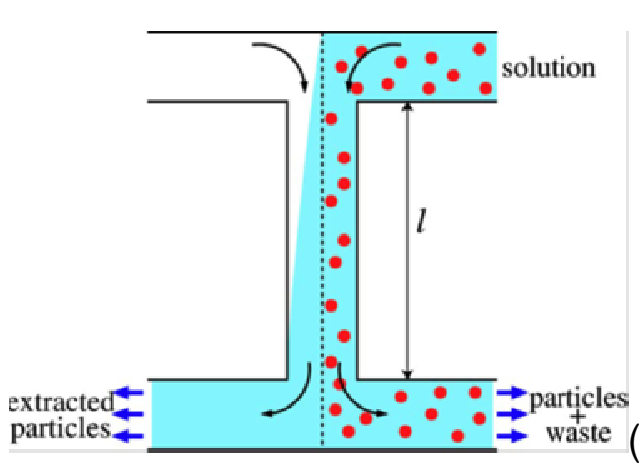

(b)

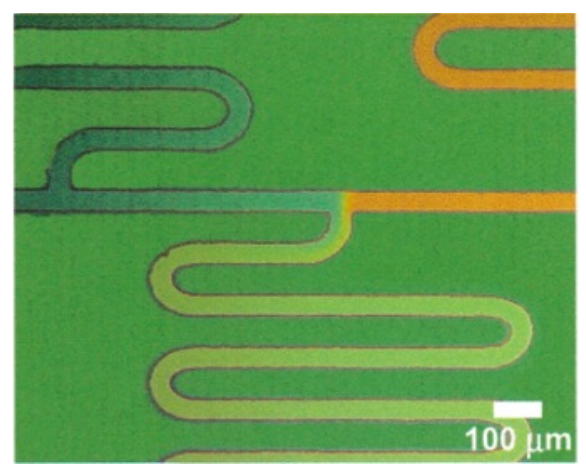

Figure 1.1.1.1. (a) This schematic illustrates the use of laminar flow and diffusivity to separate particles. ${ }^{16,20,21}$ Reprinted with permission from Review of Modern Physics, 2005, 77, 977-1026. Copyright 2005 American Physical Society. (b) This device mixes fluids using wavy channels to form a gradient. $^{22}$ Reprinted with permission from Analytical Chemistry, 2000, 15, 8311-8316. Copyright 2000 American Chemical Society.

To provide a historical context for microfluidics and microPADs it should be noted that microfluidics blossomed roughly 30 to 40 years ago and was expected to revolutionize fluid mechanics, drug testing, and clinical diagnosis much the same way the microchip has revolutionized the computational power of computers. $^{16}$ The field has fallen short of its projected glory though. ${ }^{6,11,16}$ Many of the most impressive microfluidic feats have been performed with finely fabricated devices made out of silicon and glass. ${ }^{16,17}$ These materials require specialized skill and intensive labor for fabrication and are not ideal for rapid prototyping or mass scale production. The application of conventional microfluidic devices in the real world, especially resource-poor regions, has been scarce. ${ }^{11}$ Microfluidics as 
a field strives to reach the 'lab-on-a-chip' motto, but the reality is more of a 'chipin-a-lab' situation. ${ }^{17}$ In 2007 the Whitesides Research Group at Harvard University introduced paper-based microfluidics with the idea of using these paper devices as an answer to the need for low-cost diagnostics in the developing world. ${ }^{2-4}$

\subsubsection{Lateral-Flow Assays}

Lateral-flow assays (LFAs) are an immunochemical-based method that involves a single application of a sample, which wicks by capillary action through a carrier material from a sample addition zone to a test zone. ${ }^{23}$ As the sample wicks through the carrier material, it dissolves and re-suspends chemicals stored on the device, which generate a color change in response to the presence of an analyte. The technique is based on immunochromatography, which relies on the specific interaction of an antigen and antibody to provide detection of an analyte. ${ }^{23}$ This class of devices is commonly used for detecting disease markers in samples (e.g. blood, urine or saliva) in POC testing. ${ }^{23,24} \mathrm{POC}$ is the concept of bringing clinical testing to the patient whether in a bed at a hospital, at home or in the field. LFAs are ideal for diseases or conditions that only require a yes/no answer because they are usually qualitative. A common example is the pregnancy test. Figure 1.1.2.1 show the basic steps for how an LFA functions.

Another type of immunoassay is the enzyme-linked immunosorbent assay (ELISA). ELISAs are often quantitative and are typically much more sensitive 
than LFAs because they couple the inherent specificity of antibodies with a colorimetric signal amplification system. ${ }^{23}$ These assays are also typically performed in plastic well plates instead of on porous membranes. There are many versions of ELISA, but the most basic version, called direct ELISA, is carried out in four steps: (i) first, an antigen present in a sample is immobilized onto the surface of a well, (ii) then the enzyme-linked antibody specific to the antigen is added and allowed to bind to the antigen, (iii) the well is then washed to remove unbound antibody, and (iv) an enzyme substrate is added to the well, which will be converted by the enzyme into a product, typically a colored product, that can be measured and gives a signal that is proportional to the amount of antigen present in the sample.

ELISAs are not typically performed in an LFA format because of the difficulty of automating all the steps of the assay. A potential advantage of microPADs over conventional LFAs is that the sequential reagent addition required for an ELISA could be achieved and automated with proper engineering of the device. 


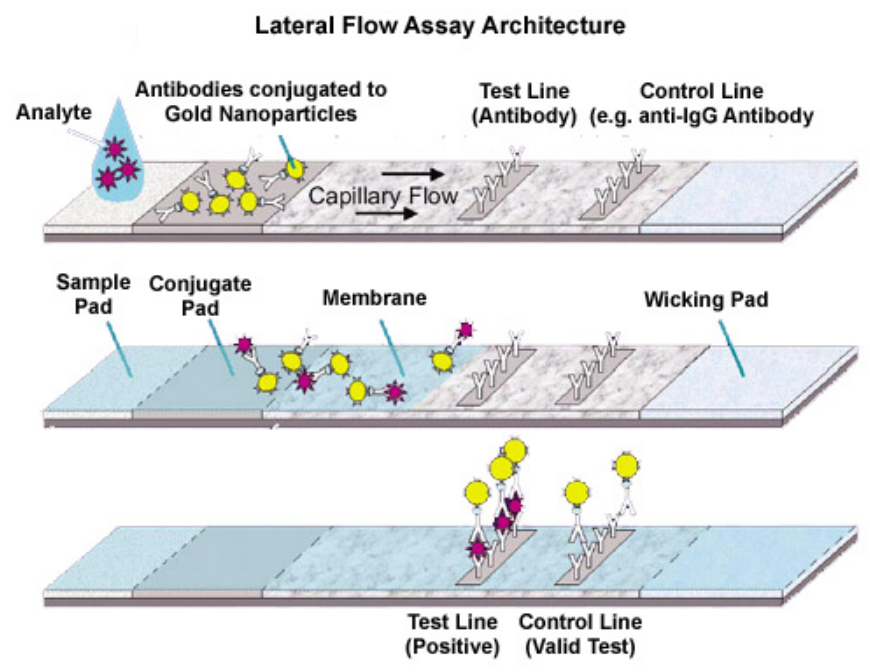

Figure 1.1.2.1. Depicted is the layout and process of a gold nanoparticle lateralflow assay showing a generic interaction between an analyte, antibodies, and antigen. ${ }^{23}$ Reprinted with permission from Eline P. Meulenberg, Chapter 11, Antibodies Applications and New Developments, Bentham E-Books.

\subsubsection{Colorimetric Assays}

Colorimetric assays produce color changes when analytes are present. The obvious signal given by colorimetric assays make them an ideal qualitative mechanism for detecting analytes (e.g. glucose, proteins, and antibodies). Many colorimetric assays are employed only to give a yes/no qualitative determination for the presence of an analyte. Colorimetric assays can also be quantitative. The color intensity for most colorimetric assays is a function ofthe concentration of the analyte in the sample. This relationship allows for external standard curves to be produced using a series of analyte solutions of known concentration. Using the best-fit line and equation of said line, it is possible to determine the 
concentration of unknown samples of the same analyte within the range of the external standard solutions. Colorimetric assays exist for a wide range of analytes and are often performed in solution or on a porous matrix like paper. MicroPADs are a suitable platform for colorimetric assays and many of the existing colorimetric tests can be adapted to be performed on microPADs.

There are two colorimetric assays that were used widely throughout this body of work for qualitative and quantitative purposes. The first is a glucose assay that involves the coupling of two enzyme-facilitated reactions. The first half of the coupled reaction, facilitated by glucose oxidase (GOX), is the conversion of D-glucose and oxygen $\left(\mathrm{O}_{2}\right)$ into D-glucono- $\delta$-lactone and hydrogen peroxide $\left(\mathrm{H}_{2} \mathrm{O}_{2}\right)$. The second half, facilitated by horseradish peroxidase (HRP), is the oxidation of 2,2'-azino-bis(3-ethylbenzothioazoline-6-sulfonic acid) (ABTS) from a

colorless reduced state to green-blue oxidized state. ${ }^{7}$ This reaction involves the transfer of electrons from ABTS to $\mathrm{H}_{2} \mathrm{O}_{2}$ while producing water $\left(\mathrm{H}_{2} \mathrm{O}\right)$ as a byproduct. In some experiments in this body of work, only the HRP-facilitated reaction was employed. This reaction required the addition of $A B T S$ and $\mathrm{H}_{2} \mathrm{O}_{2}$ and was used to confirm the presence and estimate the activity of HRP.

\subsection{MicroPAD Challenges}

Even though microPADs have come a long way since their introduction in 2007 by the Whitesides Research Group at Harvard University, there still are many challenges to be overcome before they reach their potential as a 
ubiquitous on-the-spot analytical testing platform..$^{2,4,6,9,11}$ Three significant barriers have been addressed by the research within this thesis. First is the development of a simple method for introducing reagents into microPADs in specific, sequential steps in a way that is automated and relies on minimal input from the user (Figure 1.2.1). This technology will be useful for performing tests that require multiple steps like an ELISA (Figure 1.1.2.1). Second is the development of a method for generating external calibration curves for assays at the point-ofcare. It is envisioned that microPADs will be used in the field to conduct tests. However, for the tests to be quantitative, the results must be compared to an external calibration curve. The problem is that unless the calibration standards and the sample are tested under the same conditions, the calibration curve may not be valid. ${ }^{7,26}$ An obvious way to overcome this problem is to test the external standards at the same time as the sample is tested, and this work introduces a simple way of doing so. Third is the development of methods for storing reagents for use with microPADs for extended periods of time..$^{28-30}$ The devices should have a shelf-life of at least 6 months without refrigeration if they are going to be useful in remote regions. ${ }^{2}$ Many reagents, especially enzymes, are unstable when stored in dry form on the paper-based device. ${ }^{28-32}$ Storing enzymes in an aqueous environment leads to improved shelf life, and this work introduces a convenient way of doing so. All three achievements were made possible by introducing thermally-responsive polymers as a reagent storage and delivery system for use with microPADs. 
(a)

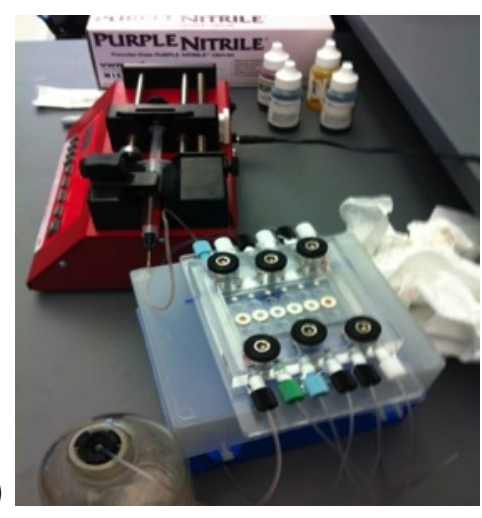

(b)

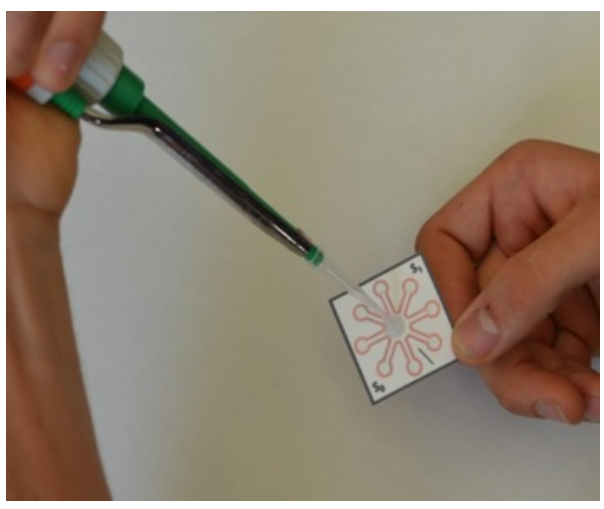

Figure 1.2.1. (a) A microfluidic device on a lab bench top using a syringe pump drive liquid through the device. (b) An end-user delivering a liquid sample to a microPAD using a micropipette.

\subsection{Introduction of Polymers}

A polymer is a large molecule, or macromolecule, which consists of hundreds or thousands of smaller molecules covalently bonded to each other. ${ }^{33}$ The small molecule is a repeating unit called a monomer. The reactions known to chemically attach monomers and build a polymer are called polymerizations. A discussion on the variety of polymerizations is beyond the scope of this work and only chain growth polymerizations will be discussed further as it is relevant to the work at hand. 


\subsubsection{Chain-Growth Polymerization}

Chain-growth polymerization is based on a chain reaction involving four steps: initiation, propagation, chain transfer, and termination. ${ }^{33}$ There are three types of active species that participate in chain-growth polymerization: radical, anionic, and cationic species. ${ }^{33}$ In radical chain polymerization, the type pertinent to this work, the initiation step involves two reactions; a homolytic cleavage of an initiator molecule followed by the addition of the resulting radical to a monomer. This monomer molecule is the chain initiating radical from which the polymer grows. Propagation is the successive addition of monomer molecules to the free radical terminus of the growing linear chain. After the addition of each monomer unit, the polymer chain's radical center transfers to the newest monomer added allowing propagation to continue. This may occur hundred to thousands of times before the polymer incurs chain transfer or terminates. Chain transfer effectively ends the propagation of the chain by transferring the radical center to another species such as monomer, another polymer chain, or a solvent molecule. The effect of chain transfer on the length of the polymer being produced is dependent on whether the rate of the reinitiation is comparable to the initial propagation rate. Termination, on the other had, is the extinction of the radical via a bimolecular reaction. Termination occurs via two mechanisms: combination and disproportionation. Combination is the bonding of two chains by the coupling of the radicals on the chains. Disproportionation is the transferring of a hydrogen beta to the radical center to another chain's radical center producing one saturated chain and one chain with a terminal alkene. Propagation will occur until 
the monomer has been consumed or all the radicals have been annihilated, either by on of the termination mechanisms, or by radical extinguishers (e.g. molecular oxygen $\left(\mathrm{O}_{2}\right)$ ).

Radical chain growth polymerization can be performed under different conditions: bulk polymerization, solution polymerization, heterogeneous polymerization, and other processes such as self-assembly or nanostructures. ${ }^{33}$ Solution polymerizations were exclusively used within the work of this thesis. Solution polymerizations involve the loading of reagents into a solvent filled container. The container is then purged of $\mathrm{O}_{2}$ and the polymerization is initiated. Since solution polymerizations are performed in a solvent, they avoid the problem of trapping excess heat from the reaction, which causes an autoacceleration. This autoacceleration causes the radical reaction to run away on its self, creating the risk of the reaction acting violently. Furthermore, solution polymerizations are favorable for industrial processes with high yield and lowcost. $^{33}$

Radical chain growth polymerization is commonly used to make long linear polymers quickly, but it can also be used to make nonlinear polymers. ${ }^{33}$ Figure 1.3.1.1 provides a list and accompanying drawings for a variety of common nonlinear topologies. ${ }^{33}$ The most pertinent of the nonlinear topologies to this work is crosslinked polymers. Crosslinking is the bridging of two separate polymer strands creating one larger macromolecule. A distinction should be drawn between crosslinker molecules and monomer molecules. Monomers used to make the linear backbone of polymers have one center of functionality, in this 
case meaning only one unsaturated carbon-carbon bond. Crosslinker molecules, on the other hand, have two centers of functionality, in this case two unsaturated carbon-carbon bonds. This allows the crosslinker molecules to have two radical centers, which in turn allows them to bond to and link two polymer strands. When conducting a radical chain growth polymerization in the presence of a high enough concentration of crosslinker molecules, each polymer strand will become crosslinked to others, thus forming a single large molecule. The ratio of monomer to crosslinker at which this occurs is known as the gel point. ${ }^{33}$ Once the gel point is reached, the physical properties of the polymer change drastically. ${ }^{33}$ In a gel, the polymer strands are bound to one another, thus reducing the space between each strand. This increase in proximity of one strand to another changes the intermolecular interactions as well as the viscoelastic properties of the polymer. ${ }^{32}$ The polymer used in this work is past its gel point creating a large permeable matrix that has unique physical properties (section 1.3.2 and 1.3.3). 


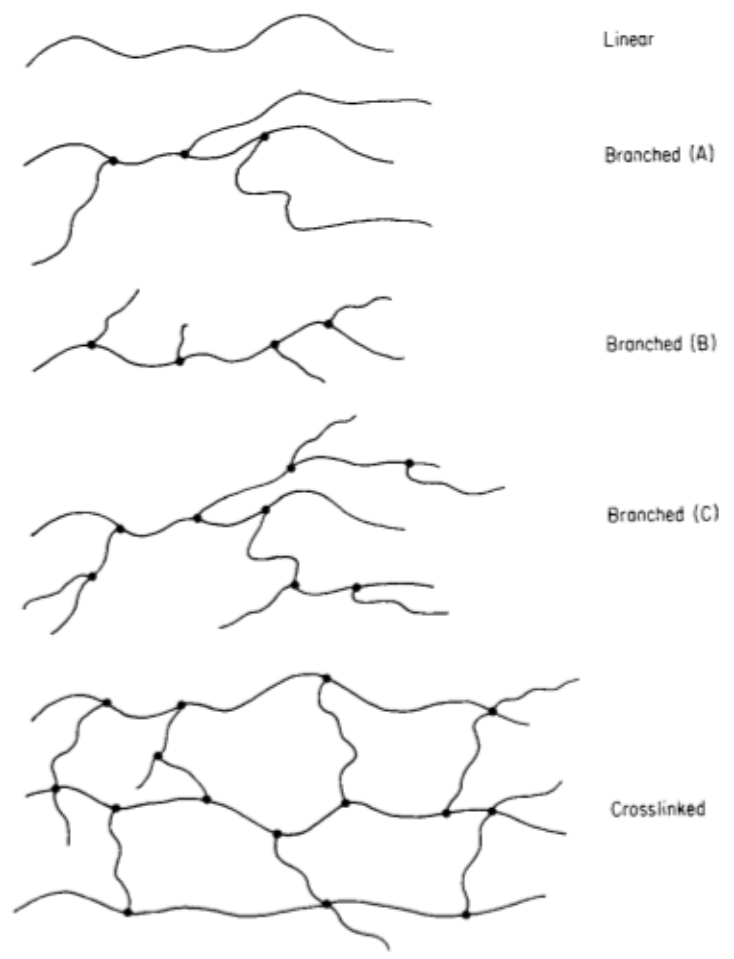

Figure 1.3.1.1. Depiction of the various polymer topologies. ${ }^{33}$ Reprinted with permission from George Odian, Principles of Polymerization, John Wiley \& Sons, Inc. $^{34}$

\subsubsection{Polymeric Gels}

Gels are unique polymer systems that consist of crosslinked polymer chains that without crosslinking would commonly be in a liquid state or semiamorphous crystalline state. ${ }^{33}$ Their extensive crosslinking creates a single large molecule that holds unique rheological properties. Most gels show thixotropic characteristics, a property in which the gel shows liquid like movement when subjected to an external force, but returns to its original shape when the external 
force is removed. ${ }^{33}$ With their unique physical and chemical properties, gels have been incorporated into numerous biomedical applications: stimulus responsive drug delivery systems, simulated semipermeable membranes, simulated muscle, prosthetics, and biocompatible batteries..$^{33,35}$ There are three major types of gels: (i) hydrogels, which are swellable in aqueous solutions; (ii) organogels, which are swellable in organic solutions; and (iii) xerogels, which are gels that have been dried without shrinkage. ${ }^{33,35}$ Hydrogels are of particular interest to this project because of their compatibility with aqueous solutions, which are typically used with microPADs. In particular, this work focused on a subclass of hydrogels, known as thermally responsive hydrogels. Thermally responsive hydrogels had the desired characteristics for this project due to their tunable physical response to a relatively simple stimulus. ${ }^{33-37}$

\subsubsection{Thermally Responsive Hydrogels}

Thermally responsive hydrogels incur conformational changes when subjected to temperature changes, and they exhibit a wide range of responses to thermal stimuli such as contraction, aggregation, and reversible gelation. ${ }^{33-37}$ The contraction and/or aggregation have an effect on the solubility of the gel, which is temperature and concentration dependent. ${ }^{35-36}$ Two major behaviors for solubility have been seen, a lower critical solution temperature (LCST) and an upper critical solution temperature (UCST). ${ }^{33,36}$ Depending on the monomer(s) used in the polymer there can exist either an LCST, a UCST or both. ${ }^{33,35}$ Monomers that 
are thermally responsive have both hydrophilic and hydrophobic moieties. The balance of the hydrophilic and hydrophobic moieties of the polymer determines whether the polymer will have a LCST or an UCST. ${ }^{32}$ An LCST occurs when a polymer is miscible with a solvent at one temperature and becomes immiscible as the temperature increases. A UCST occurs when a polymer is miscible with a solvent at one temperature and becomes miscible as the temperature increases. A general plot for the solubility profile of an LCST and a UCST can be seen in Figure 1.3.3.1.
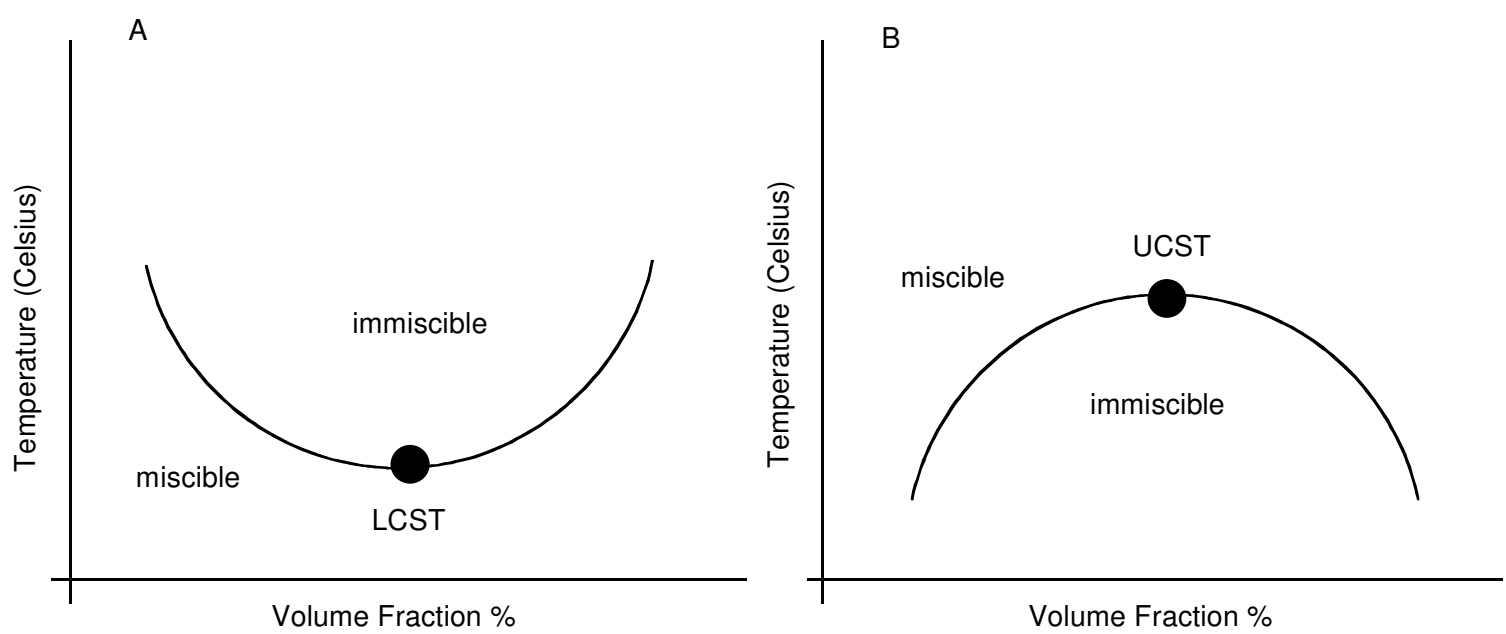

Figure 1.3.3.1. Plot of temperature versus the volume fraction of polymer in solution. UCST and LCST graphs shown for clarification of the reversible switch of solubility.

The hydrogel used throughout this work is a well-studied, thermallyresponsive hydrogel called poly(N-isopropylacrylamide) or $\mathrm{P}(\mathrm{NIPAM})$. $\mathrm{P}(\mathrm{NIPAM})$ is made from an amphipathic monomer containing a hydrophilic amide group and 
a hydrophobic isopropyl group. The LCST of P(NIPAM) is reported to be 32 ${ }^{\circ} \mathrm{C}$. ${ }^{33,36-38}$ At temperatures below $32{ }^{\circ} \mathrm{C}$, the hydrophilic amide is able to hydrogen bond with water making the polymer miscible with water. ${ }^{36}$ At temperatures above $32{ }^{\circ} \mathrm{C}$, thermal agitation causes the isopropyl group to spin and exclude the water molecules from the gel matrix making the polymer immiscible with water, and the polymer collapses into dehydrated globules (Figure 1.3.3.2). ${ }^{33,36}$ The dehydrated or chain collapsed state is further stabilized and driven thermodynamically by the favorable interaction of the carbonyl group hydrogen bonding with the hydrogen of the amide nitrogen on adjacent chains. ${ }^{38}$ Furthermore, an increase in temperature increases the influence of the entropic components of the system. ${ }^{38}$ When the water molecules trapped within the P(NIPAM) matrix are driven out, they are able to access a less ordered state. This increase in entropy also drives the expulsion of water from the gel above the LCST. $^{38}$

In general the LCST of a polymer increases when hydrophilic interactions increase and decreases when hydrophobic interactions increase. ${ }^{36}$ The LCST of thermally-responsive polymers are often affected by the presence of salts, surfactants, and acids or bases, but P(NIPAM)'s LCST is robust. ${ }^{38}$ Another interesting property of thermally responsive polymers is that the physical change they undergo in response to temperature changes is reversible. ${ }^{36}$ This property is useful in the context of this work since it can be used to load P(NIPAM) with specific analytes, which can then be delivered in a controlled manner using temperature as a stimulus. 


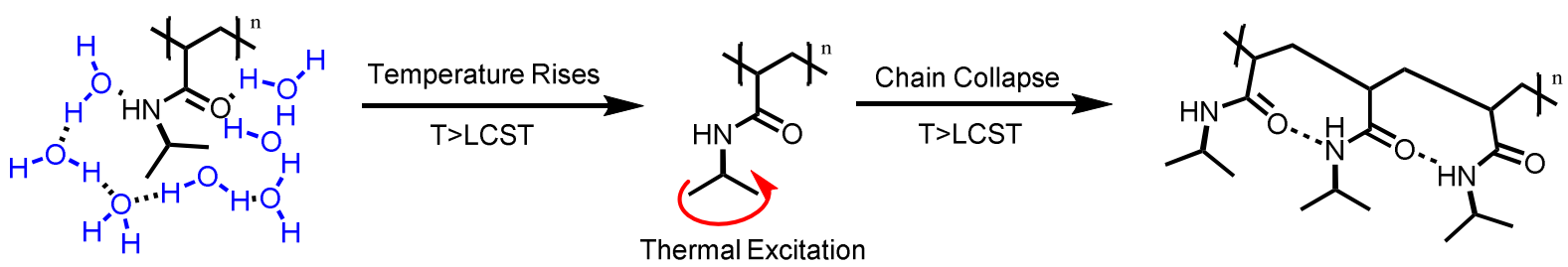

Figure 1.3.3.2. An increase in isopropyl rotation is caused by thermal excitation. The fast rotating isopropyl group disturbs the water within the hydrogel matrix and causes the chains to collapse.

\subsection{Experimental Plan and Overview}

The experimental plan guiding this work had four primary objectives: (i) optimization of the P(NIPAM) for handling and use with microPADs; (ii) characterization of the fluid-delivery from P(NIPAM) to microPADs, and the development of methods to carry out sequential as well as simultaneous reagent addition for two or more reagents; (iii) characterization of the delivery of clinically relevant analytes and reagents from $\mathrm{P}(\mathrm{NIPAM})$ to microPADs, and the development of methods for preparing external calibration curves; and (iv) characterization of the storage of reagents, specifically enzymes, in P(NIPAM) for extended periods of time. 
The investigation of P(NIPAM) started with an optimization of the synthesis. The hydrogel would be handled frequently and needed to be rugged enough to survive all the handling. The crosslinker to monomer ratio was varied to find an ideal ratio that gave a gel with the desired mechanical properties. Once synthesis conditions were decided upon, a protocol for removing the sol from the gel was explored. The sol of a gel is the vestigial reagents and unreacted monomer or crosslinker. Removal of the sol is a purification step required for producing gels that deliver consistent masses of fluid.

Next, the fluid delivery characteristics of P(NIPAM) were probed. Initial experiments showed that the surface area exposed to the heat source was the major factor affecting the mass of fluid delivered by the gel. Thus an in-situ molding technique for P(NIPAM) using poly(ethylene) straws as molds was developed in order to generate gels with consistent surface areas (Figure 1.4.1). This technique allowed for gels with reproducible dimensions to be fabricated, and thus improved the precision of the fluid delivery experiments. This work was followed by the development of methods to introduce multiple reagents from different hydrogel samples into a single microPAD. 


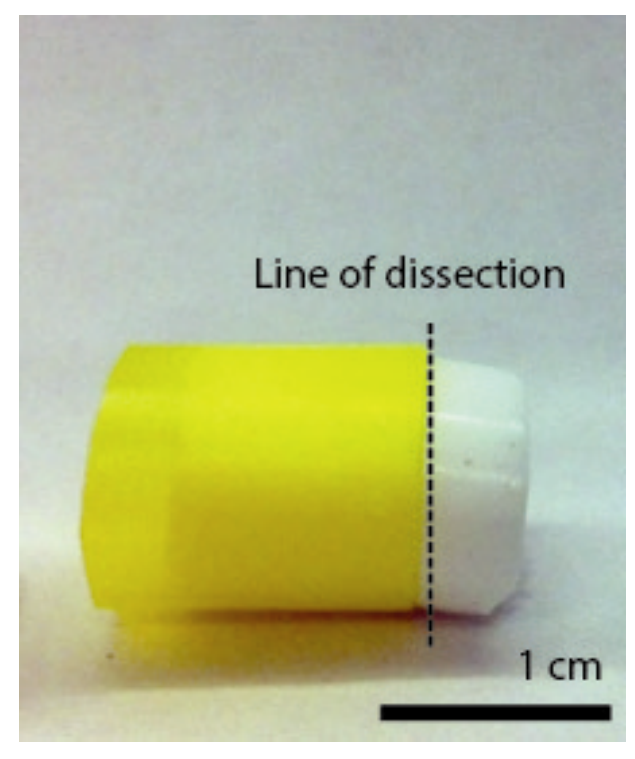

Figure 1.4.1. The dotted line indicates the path of dissection for a discrete sample. The P(NIPAM) sample show has a $11.03 \mathrm{~mm}$ diameter.

Once the fluid-delivery of P(NIPAM) to microPADs was characterized, the ability of $\mathrm{P}($ NIPAM) to deliver specific reagents to microPADs was assessed. This work focused on four major classes of reagents relevant to point-of-care diagnostics: water-soluble small molecules, enzymes, antibodies and DNA. There were three primary goals for this portion of the project: (i) to determine if P(NIPAM) was able to deliver the reagent to microPADs, (ii) to determine if P(NIPAM) had any effect on the concentration (or activity, in the case of the enzyme) of the reagent it was delivering, and (iii) to determine if P(NIPAM) could deliver the reagent accurately and precisely enough to construct an external calibration curve for assays. 
Finally, P(NIPAM) was assessed as a storage system for enzymes. The goal of this portion of the project was to study the effects, if any, of storing enzymes in P(NIPAM) for extended periods of time, and then delivering them to microPADs. 


\section{EXPERIMENTAL}

\subsection{Chemicals and Materials Used}

All reagents were purchased from commercial sources unless otherwise stated. The following chemicals were used: N-isopropylacrylamide (NIPAM, Sigma Aldrich), $N, N^{\prime}$-methylenebisacrylamide (BIS, Fisher Bioreagents), potassium persulfate (KPS, Sigma Aldrich), tetramethylethylenediamine (TEMED, Fisher Bioreagents), food color (Durkee), Glucose (Sigma Aldrich), horseradish peroxidase (HRP, MP Biomedical), glucose oxidase (GOX, MP Biomedical), IgG from Mouse Serum (Sigma Aldrich), fluorescein tagged DNA, a 20 base pair-long oligonucleotide (Biosearch Technologies), fluorescein tagged rabbit anti-sheep serum (Thermo Scientific), 2,2'-azino-bis(3ethylbenzothioazoline-6-sulfonic acid) diammonium salt (ABTS, Alfa Aesar), 1Step ${ }^{T M}$ ABTS (a proprietary solution of ABTS and $\mathrm{H}_{2} \mathrm{O}_{2}$, Thermo Scientific), and 98\% hydrogen peroxide $\left(\mathrm{H}_{2} \mathrm{O}_{2}\right)$ (Fisher Scientific). A buffer solution, $1 \mathrm{X}$ phosphate buffer saline solution (1XPBS) was prepared from 10XPBS (Fisher Scientific) with in-house nanopure water obtained from a nanopure dispenser (Thermo Scientific D13661).

The following materials were obtained from commercially available sources and were used in the experiments: chromatography paper (Whatman No. 1), thermal laminating pouches (3M, Scotch) and pressure sensitive pouches (3M, Scotch). poly(ethylene) (PE) straws of three diameters: $11.03 \mathrm{~mm}$ (Karat), $7.24 \mathrm{~mm}$ (Crystal Ware), and $5.35 \mathrm{~mm}$ (Starbucks Coffee Company). 


\subsection{Fabrication of MicroPADs}

\subsubsection{Wax Printing}

MicroPADs were fabricated by the method of wax printing. ${ }^{13}$ The pattern for the microPAD was drawn up in CleWin or AutoCAD. Then using a solid-ink printer (Xerox Phaser 8560), wax lines were printed onto chromatography paper. The paper was then baked in a convection oven (MTI Compact Forced Air) for 2 minutes at $195{ }^{\circ} \mathrm{C}$ to melt the wax and create hydrophobic barriers. An alternative baking process involved heating the paper for 15 minutes at $145{ }^{\circ} \mathrm{C}$. The alternative baking process was used for fine $(200 \mu \mathrm{m})$ wax line to reduce leaks. The paper was then cooled to room temperature under ambient conditions. The paper was stored in square petri dishes (Fisher Scientific), until it was used as an assay platform.

\subsubsection{Lamination of MicroPADs}

Once microPADs were fabricated by wax printing, the channel networks could be enclosed with lamination sheets. Enclosing the devices was advantageous because it protected the channels from contamination and minimized evaporation of fluids from the channels. Two different methods for lamination were used: a hot lamination and a cold or pressure-sensitive lamination. The hot lamination was performed by inserting the microPAD in a thermal laminating pouch and running the pouch through a thermal laminator (APACHE AL-12P) at $250{ }^{\circ} \mathrm{F}$. The cold or pressure-sensitive lamination was 
performed by placing the microPAD in a pressure-laminating pouch. The pouch was then sealed by either running it through the thermal laminator set to $0{ }^{\circ} \mathrm{F}$ (room temperature) or manually pressing it onto the microPAD.

Hot or cold lamination was used depending on the device requirements. For some devices, a combination of hot and cold lamination was used. In these cases, the hot lamination was performed first on the top face of the microPAD, reagents were then added and dried on the device, and, finally, the bottom face of the device was sealed using cold lamination. This procedure ensured that the reagents, some of which were thermally sensitive, did not have to be subjected to the elevated temperatures of the thermal laminator required for hot lamination. Other devices used only a single sheet of hot or cold lamination pouches.

\subsection{ImageJ Quantitative Analysis}

The results from colorimetric assays conducted on microPADs were imaged using a scanner (Epson Perfection V300) with the following conditions: 48-bit Color image type, 300dpi resolution, and reflective document type. Images were analyzed using ImageJ $1.46 \mathrm{r}$, open source analysis software. First the device was scanned and cropped to ensure it was of an appropriate file size manageable by ImageJ. Once opened in ImageJ, the image was split into three color-channels; red, green and blue. The green and blue channels were discarded, and the red channel was then inverted. This process reduces background noise and thus decreases indeterminate error. From the inverted red 
channel image, the color intensity of the test zone was measured using a microarray profile plugin. ${ }^{39}$ The most variable component of the image analysis was the diameter used for the circles of the microarray profile plugin. The diameter of the microarray profile plugin circles ranged from 10 to 75 units. The color intensity values obtained were analyzed in Excel. This protocol was followed unless otherwise stated.

\subsection{Synthesis and Handling of Poly(N-isopropylacrylamide)}

\subsubsection{Synthesis of Poly( $\mathrm{N}$-isopropylacrylamide)}

Poly(N-isopropylacrylamide) (P(NIPAM)) was synthesized via free radical solution polymerization as seen in Scheme 2.4.1.1. The synthesis was performed at room temperature using three purged vials. Molar ratios of monomer to crosslinker of $1: 1,3: 1,4: 1,5: 1,6: 1,7: 1,8: 1$, and 10:1 were tested. The following procedure is described for a monomer to crosslinker molar ratio of $5: 1$, which was used for all experiments with microPADs. Vial 1 contained NIPAM $(1.209 \mathrm{~g}$, 10.68 mmoles) and $\mathrm{BIS}\left(0.3294 \mathrm{~g}, 2.14\right.$ mmoles) in $\mathrm{DI}_{2} \mathrm{O}(15 \mathrm{~mL})$. Vial 2 contained the KPS $(0.009 \mathrm{~g}, 0.03$ mmoles $)$, radical initiator, in $\mathrm{DI}_{2} \mathrm{O}(2.0 \mathrm{~mL})$. Vial 3 contained TEMED (71 $\mu \mathrm{L}, 0.06 \mathrm{~g}, 0.47 \mathrm{mmoles})$, a radical generator accelerator, in $\mathrm{DI} \mathrm{H}_{2} \mathrm{O}(2.0 \mathrm{~mL})$. Each vial was capped with a rubber septum, which was fastened with copper wire and then purged with nitrogen gas $\left(N_{2}\right)$ for 20 minutes under atmospheric pressure. After purging, the contents of Vials 2 and 3 were added to Vial 1 . Vial 1 was then taken off the nitrogen purge line, 
shaken thoroughly and allowed to react to completion at room temperature for 24 hours. The polymerization was considered to be complete when an opaque gel had solidified in Vial 1. This occurred within 10 minutes of combining the contents of of the three vials. The gel was left over night to ensure the polymerization had gone to completion.

Scheme 2.4.1.1. Synthetic plan for poly(N-isopropylacrylamide).

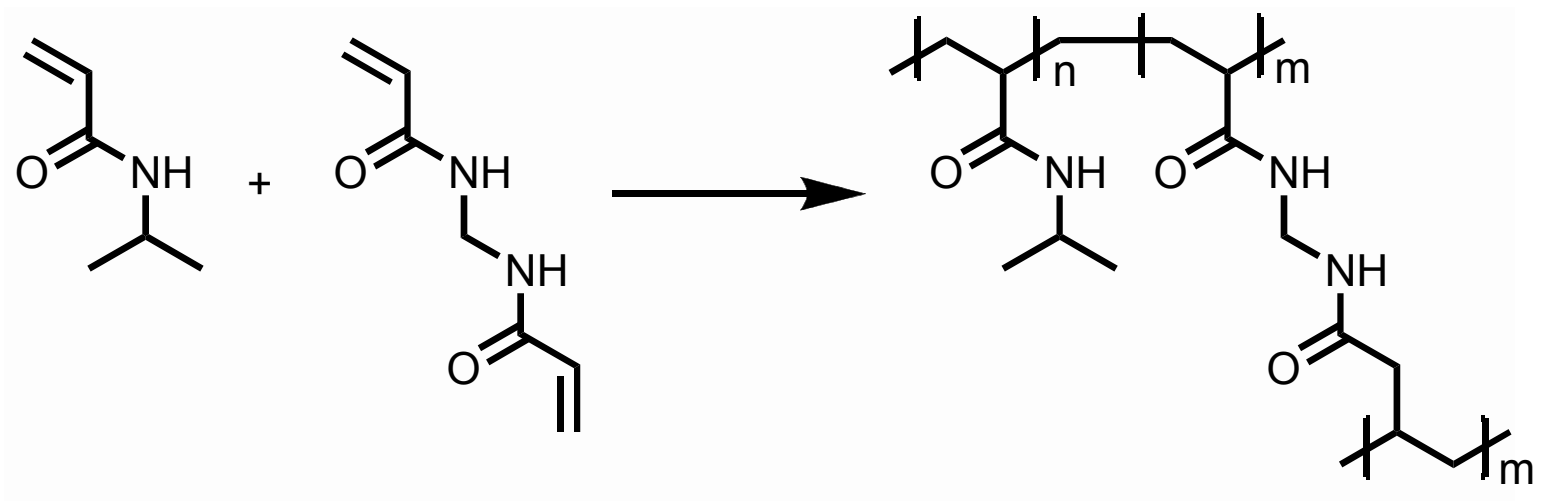

Conditions: NIPAM, BIS, TEMED, KPS, DI $\mathrm{H}_{2} \mathrm{O}, \mathrm{RT}$

In order to create samples of P(NIPAM) with a defined surface area, PE straws with diameters of $5.37 \mathrm{~mm}, 7.24 \mathrm{~mm}$, and $11.03 \mathrm{~mm}$ were used as inexpensive molds for P(NIPAM). If samples of particular diameters were desired the straws were placed into Vial 1 prior to purging and retrieved with molded P(NIPAM) once the reaction was complete. To produce varying lengths of sample, the length of the straws were varied as well as the size of the vial. Regardless of the size of the vial, molar ratios of the reagents were kept constant 
(i.e., NIPAM (5): BIS (1): TEMED (0.22): KPS (0.016)) and solvent volumes were scaled proportionally.

\subsubsection{Isolation of P(NIPAM)}

After 24 hours, the reaction vial (Vial 1) was etched with a glasscutter immediately above the P(NIPAM). Once the circumference was thoroughly etched, the rubber septum was removed, and the vial was immersed in a liquid nitrogen bath for approximately 45 seconds, ensuring that the entire etched path was exposed to liquid nitrogen. Immediately after liquid nitrogen exposure, the vial was shattered along the etch line using channel-lock pliers. The vial was covered with an aluminum foil sheet to contain the shattered glass. The strawencased $\mathrm{P}($ NIPAM) hydrogel samples were retrieved from the P(NIPAM) mass and then thermocycled to expel unreacted residual reactants.

\subsubsection{Hydrogel Sol Removal Via Thermocycling}

Samples were thermocycled after retrieval in order to purify the P(NIPAM) hydrogels by ridding the polymer matrix of any residual reagents. The thermocycling procedure involved the following steps: i) submerge the P(NIPAM) in $\mathrm{DI} \mathrm{H}_{2} \mathrm{O}$ and heat for 10 minutes at $60{ }^{\circ} \mathrm{C}$ in a silicon oil bath, ii) decant the water and rinse the samples in the vial with $\mathrm{DI} \mathrm{H}_{2} \mathrm{O}$ two times, iii) refill the vial with $\mathrm{DI} \mathrm{H}_{2} \mathrm{O}$ and place it in an ice-water bath $\left(0^{\circ} \mathrm{C}\right)$ for 20 minutes, iv) remove 
the vial of P(NIPAM) and allow to warm to room temperature. The cycle was repeated a total of 6 times for each hydrogel.

To assess whether the sol had been removed from the gel, three samples of comparable mass for each diameter $(5.35 \mathrm{~mm}, 7.24 \mathrm{~mm}$, and $11.03 \mathrm{~mm})$ were weighed before and after being exposed to $60{ }^{\circ} \mathrm{C}$ for 10 minutes for each thermocycle. The mass of the samples averaged $0.13 \mathrm{~g}, 0.15 \mathrm{~g}$, and $0.35 \mathrm{~g}$ for diameters of $5.35 \mathrm{~mm}, 7.24 \mathrm{~mm}$, and $11.03 \mathrm{~mm}$, respectively.

\subsubsection{Characterization of P(NIPAM) Fluid Delivery}

Samples of P(NIPAM) of three diameters, $(5.35 \mathrm{~mm}, 7.24 \mathrm{~mm}$, and 11.03 $\mathrm{mm}$ ) were used to study the fluid delivery characteristics of the hydrogel at temperatures above the LCST as a function of the sample's mass and time. For assessing the fluid delivery characteristics as a function of mass, P(NIPAM) samples of each diameter were prepared by dissecting portions of the hydrogel using a razor blade. Samples were produced in mass ranges of 0.0229 to $1.9178 \mathrm{~g}, 0.0776$ to $3.0743 \mathrm{~g}$ and 0.2241 to $8.2954 \mathrm{~g}$, for the diameters of 5.35 $\mathrm{mm}, 7.24 \mathrm{~mm}$ and $11.03 \mathrm{~mm}$ respectively. Each sample was weighed and then placed on a digital hot plate (Thermo Scientific MAREC) at $40{ }^{\circ} \mathrm{C}$ for 12 minutes. The sample was weighed again after the heating step. The difference of the initial and final mass of the P(NIPAM) hydrogel was used to determine the mass of fluid delivered. 
To study the effect of the duration of the heat exposure on the amount of fluid delivered by P(NIPAM), two samples for diameters $5.35 \mathrm{~mm}$ (with initial masses of $0.767 \mathrm{~g}$ and $1.75 \mathrm{~g}$ ), $7.24 \mathrm{~mm}$ (with initial masses of $1.25 \mathrm{~g}$ and 3.49 $\mathrm{g}$ ), and $11.03 \mathrm{~mm}$ diameter gel (with initial masses $1.56 \mathrm{~g}$ and $6.12 \mathrm{~g}$ ) were prepared. All prepared samples were reused throughout the series of time trials. Samples were weighed prior to the time trial, then heated at $40{ }^{\circ} \mathrm{C}$ on a digital hot plate for a specified time ranging from 2 to 60 minutes, and weighed after exposure. Then, the samples were cooled and rehydrated in a room temperature DI $\mathrm{H}_{2} \mathrm{O}$ bath for 1 hour before being reused.

\subsubsection{Loading Reagents into P(NIPAM)}

To load reagents or dyes into the hydrogel, the gel was first thermally excited to expel all fluid, then transferred to a vial with the desired loading solution and allowed to equilibrate. The gel was thermally excited by submerging it into $\mathrm{DI} \mathrm{H}_{2} \mathrm{O}$ in glass vials and then placed into a silicone oil bath for heating. The initial temperature was $60{ }^{\circ} \mathrm{C}$, and the temperature was increased by $10{ }^{\circ} \mathrm{C}$ every 30 minutes from 60 to $80{ }^{\circ} \mathrm{C}$. Once the silicone bath reached $80{ }^{\circ} \mathrm{C}$, the hydrogels were heated for an additional 30 minutes. The samples were then transferred to vials containing analyte solutions that were at room temperature $\left(23^{\circ} \mathrm{C}\right)$ and allowed to equilibrate for 24 hours. All P(NIPAM) samples were loaded in this manner unless otherwise stated. 


\subsection{Application of P(NIPAM) to MicroPADs}

\subsubsection{Preliminary Fluid Delivery Assessment of P(NIPAM)}

P(NIPAM) was loaded with aqueous dye solutions and placed on various microPADs to study fluid delivery on paper. The P(NIPAM) samples were soaked in $50 \% \mathrm{v} / \mathrm{v}$ food color and $\mathrm{DI} \mathrm{H}_{2} \mathrm{O}$ solutions for 24 hours before being used with microPADs. One microPAD was used to measure the rate of wicking for fluids delivered to paper-based devices via P(NIPAM). The microPAD used to measure the wicking rate of fluid delivered via P(NIPAM) consisted of a single circular sample addition zone with a diameter of $7.3 \mathrm{~mm}$ leading into a 6 -cm-long and 2mm-wide channel with markings every centimeter along both sides of the channel, which let out into a waste zone. A second microPAD was used to demonstrate that P(NIPAM) could effectively deliver fluids in a specific sequence. The microPAD used to demonstrate sequential addition of fluids via P(NIPAM) had three staggered circular sample addition zones all with a diameter of $7.3 \mathrm{~mm}$ leading into a single channel that led to a waste zone. A third microPAD was used to demonstrate that $\mathrm{P}(\mathrm{NIPAM})$ could deliver fluids in laminar flow. The microPAD used to demonstrate laminar flow had three circular sample addition zones all with a diameter of $7.3 \mathrm{~mm}$ that were situated to one side of the device and were equidistant from the point of entry into a single channel that let out into a waste zone. All three devices were laminated using thermal lamination pouches. The lamination over the sample addition and waste zones was cut out and removed to allow for sample introduction and fluids to evaporate in the waste 
zone. The cutting of the lamination layer was performed manually with a razor blade.

\subsubsection{Assessment of P(NIPAM) as a Delivery System for Clinically Relevant} Assays

P(NIPAM) was used to deliver four distinct, clinically-relevant classes of analytes: small molecules (glucose), enzymes (HRP), antibodies (fluoresceinlabeled IgG from rabbit) and DNA (fluorescein-labeled 20 base pair oligonucleotide). The analytes were loaded into P(NIPAM) via thermocycling.

\subsubsection{Delivery of Glucose}

$\mathrm{P}$ (NIPAM) was demonstrated to deliver glucose in a quantitative manner to microPADs by two experiments. Both experiments employed a paper-based 96-well plate with circular reaction zones with a diameter of $5 \mathrm{~mm}$. All P(NIPAM) samples used in this experiment were synthesized in the same batch. P(NIPAM) samples with a $7.24 \mathrm{~mm}$ diameter and length of approximately $4 \mathrm{~cm}$ were prepared for loading with the glucose solutions by thermocycling. All glucose solutions were prepared using glucose in $\mathrm{DI} \mathrm{H}_{2} \mathrm{O}$. The concentration of glucose delivered was quantified using a colorimetric glucose assay based on a coupled enzymatic reaction using GOX, HRP, glucose and ABTS, which produced a blue- 
green color. A reaction mixture was prepared consisting of GOX $\left(75 \mathrm{kU} \mathrm{L}^{-1}\right)$, HRP $\left(250 \mathrm{kU} \mathrm{L}^{-1}\right)$ and ABTS $(6.25 \mathrm{mM})$ in 1XPBS.

Glucose solutions of the following concentrations were prepared in duplicate: $0 \mathrm{mM}\left(\mathrm{DI} \mathrm{H} \mathrm{H}_{2} \mathrm{O}\right), 1 \mathrm{mM}, 2 \mathrm{mM}, 2.5 \mathrm{mM}, 3 \mathrm{mM}, 3.5 \mathrm{mM}, 4 \mathrm{mM}$, and 5 $\mathrm{mM}$. One series of the glucose solutions was loaded into P(NIPAM). The other set was used as external solutions for comparison. Ultimately, three different types of glucose samples were prepared: glucose loaded into P(NIPAM) (Condition 1), the excess glucose solutions remaining in the vials used to load the P(NIPAM) gels (Condition 2), and the external glucose solutions (Condition 3). A volume of $1 \mu \mathrm{L}$ of reaction mixture was spotted in the reactions zones of paper-based 96-well plate. Before the reaction mixture dried the P(NIPAM) samples (Condition 1) were placed in the wells for 60 seconds. For the P(NIPAM) loading glucose solution (Condition 2) and external glucose solutions (Condition 3), $3 \mu \mathrm{L}$ for each glucose concentration was added to the reaction zone before the reaction mixture dried. Three trials were run for each concentration of Conditions 1-3. The colorimetric assays were allowed to develop and dry for 30 minutes before being imaged and analyzed.

\subsubsection{Delivery of Fluorescein-labeled IgG}

The device used for both the delivery of fluorescein tagged antibody and DNA had a circular sample addition zone and a circular test zone, both with a 
diameter of $7.25 \mathrm{~mm}$. These zones were connected by a single channel (length 2 $\mathrm{mm}$ and width $2.2 \mathrm{~mm}$ ).

A series of dilutions of fluorescein-labeled rabbit anti-sheep antibody were prepared in duplicate in 1XPBS in concentrations of; $0.75 \mathrm{mg} / \mathrm{mL}, 0.1875 \mathrm{mg} / \mathrm{mL}$, $0.0234 \mathrm{mg} / \mathrm{mL}$, and $0.0014 \mathrm{mg} / \mathrm{mL}$. One set was loaded into $7.24 \mathrm{~mm}$ P(NIPAM) samples with an average mass of $0.1504 \mathrm{~g}$ and the other set was used as external solutions for comparison. Two other solutions, $0.2500 \mathrm{mg} / \mathrm{mL}$ and $0.0625 \mathrm{mg} / \mathrm{mL}$, were prepared to be used as unknown sample solutions.

The P(NIPAM) samples were placed upon the sample addition zone until the loaded solution wicked across the channel and completely filled the test zone. Not all of the samples successfully filled the devices. The P(NIPAM) samples were then removed from the sample addition zone and disposed of. The external solution assays were tested by adding $10 \mu \mathrm{L}$ of solution to the sample addition zone. All devices were dried for 20 minutes. Once devices were dry, they were wrapped in aluminum foil to protect the fluorescein from ultraviolet radiation. The devices were then imaged using a fluorescein scanner (Typhoon TRIO+, Amersham Biosciences) and quantified in ImageJ using a microarray profile plugin with circle diameters of 10 units. The devices were measured at the neck of the channel leading to the test zone. This was done because some devices did not receive enough fluid from the P(NIPAM) to fill the test zone. The images were measured in grey scale. 


\subsubsection{Delivery of Fluorescein-labeled DNA}

A series of dilutions of fluorescein tagged DNA (20 base-pair-long oligonucleotide) were prepared in duplicate in 1XPBS with concentrations of $7.953 \mathrm{nmol} / \mathrm{mL}, 3.976 \mathrm{nmol} / \mathrm{mL}, 1.988 \mathrm{nmol} / \mathrm{mL}$, and $0.9941 \mathrm{nmol} / \mathrm{mL}$. One set was loaded into $7.24 \mathrm{~mm}$ P(NIPAM) samples with an average mass of $0.1389 \mathrm{~g}$, and the other set was as external solutions for comparison. Two additional solutions, with concentrations of $3.635 \mathrm{nmol} / \mathrm{mL}$ and $0.9089 \mathrm{nmol} / \mathrm{mL}$, were prepared to be used as unknown sample solutions. Samples were tested by the same method as the fluorescein-labeled IgG samples. Several of the devices were not completely filled for this experiment as well. Samples were then processed via the same method as the fluorescein-labeled lgG samples.

\subsubsection{Delivery of HRP}

P(NIPAM) was assessed for its ability to deliver an enzyme, HRP, by passive wicking to a microPAD, using a paper-based 96-well plate. The effects of $\mathrm{P}$ (NIPAM) on the activity of HRP was also investigated. All P(NIPAM) samples used in this experiment were synthesized in the same batch. P(NIPAM) samples with a $7.24 \mathrm{~mm}$ diameter and length of approximately $4 \mathrm{~cm}$ were prepared for loading with the stock HRP solution. A $50 \mathrm{~mL}$ volume of HRP stock solution was prepared with a concentration of $550 \mathrm{kU} \mathrm{L}^{-1}$ in 1XPBS. Approximately $20 \mathrm{~mL}$ of the HRP stock solution was poured into a vial, which was then loaded with eight thermocycled $\mathrm{P}(\mathrm{NIPAM})$ samples and left to equilibrate for two hours in a 
refrigerator $\left(5-8^{\circ} \mathrm{C}\right)$. Another set of four thermocycled P(NIPAM) samples were loaded with 1XPBS and left to equilibrate for two hours in a refrigerator $(5-8$ $\left.{ }^{\circ} \mathrm{C}\right)$. The 1XPBS loaded P(NIPAM) samples would account for the background signal (P(NIPAM) Control). The remaining $30 \mathrm{~mL}$ of the HRP stock solution (External Solution) was stored in a refrigerator $\left(5-8{ }^{\circ} \mathrm{C}\right)$ alongside the $\mathrm{P}(\mathrm{NIPAM})$ samples. The remainder of the stock solution was stored in a refrigerator $(5-8$ $\left.{ }^{\circ} \mathrm{C}\right)$ for two hours while the $\mathrm{P}(\mathrm{NIPAM})$ samples were equilibrated. After 2 hours the P(NIPAM) samples in the stock HRP solution were then divided into two groups; half were stored in a dry, empty vial (Nonsolution Storage), and the other group was stored in $20 \mathrm{~mL}$ of stock HRP solution (Solution Storage). Nonsolution Storage, Solution Storage, and the External Solution were then stored in a drawer at room temperature $\left(23^{\circ} \mathrm{C}\right)$ for two hours before being used.

Nonsolution Storage, Solution Storage, P(NIPAM) Control, and External Solution were applied to a paper-based 96-well plate. Three samples, $3 \mathrm{~mm}$ in height, of P(NIPAM) were cut from the Nonsolution Storage, Solution Storage, and P(NIPAM) Control samples. The P(NIPAM) samples were then placed in their respective reaction zones of the paper-based 96-well plate for 10 minutes to ensure the delivery of HRP. After the P(NIPAM) samples were removed three trials of $1 \mu \mathrm{L}$ of the External Solution were added to separate reaction zones. Before any of the HRP deposited solutions dried, $3 \mu \mathrm{L}$ of 1 Step ABTS was added to develop the reaction zones. The paper was allowed to develop and dry for 30 minutes, and then the results were imaged and quantified. 
As an additional experiment it was assessed if a single sample of P(NIPAM) could deliver comparable concentrations of HRP repeatedly. After the initial ability of P(NIPAM) to deliver HRP to microPADs was confirmed the three P(NIPAM) samples from the three conditions (Nonsolution Storage, Solution Storage, and P(NIPAM) Control) were reused three more times. The samples were set in a reaction zone for 10 minutes for each use. After the 10 minutes the samples were transferred from the previous reaction zone directly to a new reaction zone. Immediately after transferring the samples, $1 \mu \mathrm{L}$ of the External Solution was added to three separate reaction zones. Each trial was developed and processed in the same manner as the initial test.

\subsubsection{Assessment of P(NIPAM) as a Storage System for HRP}

Using the P(NIPAM) samples prepared as described in section 2.5.2.2 a shelf-life experiment was performed over a span of 35 days. The Nonsolution Storage, Solution Storage, P(NIAM) Control, and External Solution were stored at room temperature $\left(23^{\circ} \mathrm{C}\right)$ in a drawer to reduce exposure to sunlight and minimize evaporation over the 35-day trail. The External Solution, stored in a drawer, was used as a control for the amount of activity of the enzyme throughout the shelf life experiment.

Three samples were cut and tested for each of the three P(NIPAM) loaded conditions (Nonsolution Storage, Solution Storage and P(NIAM) Control) for a total of nine P(NIPAM) samples each day. The samples were left on their 
designated reaction zones for 10 minutes to ensure enzyme delivery. Once P(NIPAM) samples were removed, and before the deposited solution dried, $1 \mu \mathrm{L}$ of the stock HRP solution was added to their own respective reaction zone. Then $3 \mu \mathrm{L}$ of 1 Step ABTS reagent was added to every reaction zone before the deposited solution dried. The reaction zones were allowed to develop for 30 minutes, then imaged and quantified. 


\section{RESULTS AND DISCUSSION}

\subsection{Poly(N-isopropylacrylaimide) Synthesis, Thermocycling and}

Characterization

The poly(N-isopropylacrylaimide) (P(NIPAM)) synthesis was optimized to provide a hydrogel which could be handled easily. It was determined that a monomer to crosslinker molar ratio of $5: 1$ was optimal for producing P(NIPAM) that was easy to handle and cut. The gels with ratios greater than 5:1 were too soft to handle effectively, while the gels with a lower ratio were too brittle. The ability to handle the material was crucial in this project, because it allowed for the gels to be cut into discrete masses efficiently, and for the segments to be manipulated without losing mass. Any loss of mass during handling would cause significant variations in the results. The 5:1 ratio was used throughout the work described herein.

The use of straws proved to be an effective method of molding P(NIPAM) into cylinders of reproducible diameters (Figure 3.1.1). We chose to work with straws that were commercially available, but this method of synthesis could presumably be used to prepare P(NIPAM) samples in a variety of shapes as long as the appropriate plastic casing was available. 
(a)

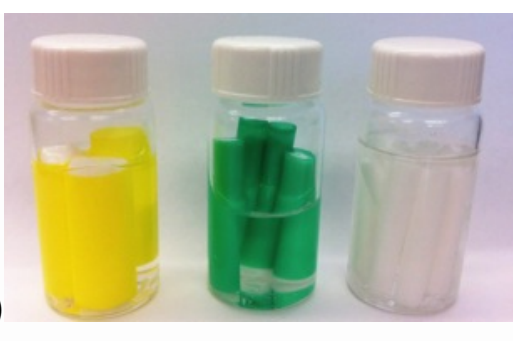

(b)

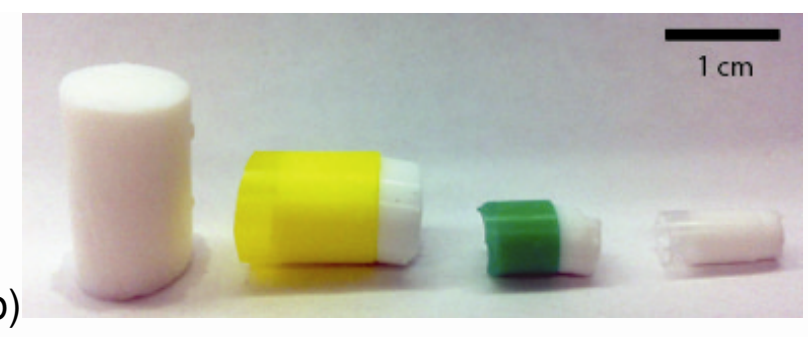

Figure 3.1.1. P(NIPAM) samples molded via straws to illustrate the array of P(NIPAM) diameters available, and the scale of the gels after synthesis and processing. (a) Three reaction vials containing the three P(NIPAM) diameters of $11.03 \mathrm{~mm}, 7.24 \mathrm{~mm}$, and $5.35 \mathrm{~mm}$ respectively, stored in $\mathrm{DI} \mathrm{H}_{2} \mathrm{O}$. (b) An unsheathed P(NIPAM) sample with a diameter of $11.03 \mathrm{~mm}$ followed by sheathed samples of P(NIPAM) with diameters of $11.03 \mathrm{~mm}, 7.24 \mathrm{~mm}$, and 5.35 $\mathrm{mm}$ respectively.

Thermocycle trends for all three diameters of P(NIPAM) are shown in Figure 3.1.2. Thermocycling the $\mathrm{P}(\mathrm{NIPAM})$ is paramount for achieving a hydrogel that delivers consistent masses of fluid. The variations in the mass percent delivered within the first four thermocycles were due most likely to expulsion of the sol from the hydrogel. The leveling off of the mass percent delivered after four cycles suggests the completion of sol expulsion. It is interesting to note that all three diameters of $\mathrm{P}(\mathrm{NIPAM})$ delivered approximately $15 \%$ of their initial mass as fluid. This occurrence is coincidental, but can be explained. Figure 3.1.3, shows that to expel $15 \%$ of the initial mass of a hydrogel with diameters of 5.35 $\mathrm{mm}, 7.24 \mathrm{~mm}$ and $11.03 \mathrm{~mm}$, one would require masses of P(NIPAM) of approximately $0.15 \mathrm{~g}, 0.25 \mathrm{~g}$ and $0.40 \mathrm{~g}$, respectively. The average masses for the $5.35 \mathrm{~mm}, 7.24 \mathrm{~mm}$ and $11.03 \mathrm{~mm}$ diameter thermocycled samples were 0.13 
$\mathrm{g}, 0.15 \mathrm{~g}$ and $0.32 \mathrm{~g}$ respectively, which are close to the ideal values. Larger surface area samples would inherently incur larger differences in mass and so smaller masses were required. Large standard deviations were observed in the mass percent delivered for all three diameters, which can be attributed to the relatively large variation in the mass of the samples used for the experiment, up to $10 \%$ standard deviation. The variation in the masses of the samples comes from the difficulty of cutting reproducible sample masses by hand. When small sample sizes are cut for assessment, differences in the masses of these small sample sizes caused large relative differences in the mass of fluid expelled when the thermally excited chains collapsed. This was a problem we faced when working with $\mathrm{P}(\mathrm{NIPAM})$ throughout this project. Ultimately, thermocycling is necessary for quality control, but is difficult to assess quantitatively. 


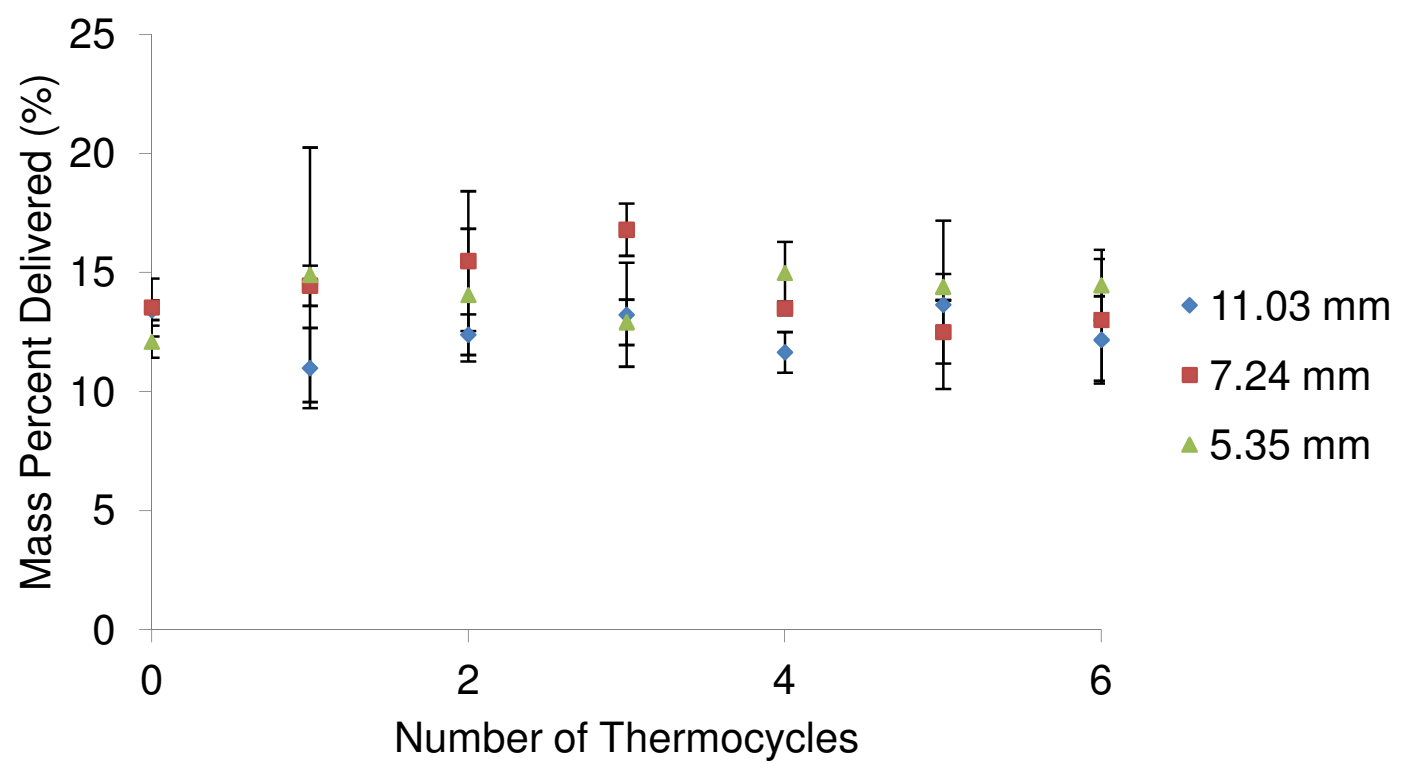

Figure 3.1.2. Plot of the mass percent expelled versus the number of thermocycles for all three diameters of $\mathrm{P}(\mathrm{NIPAM})$. Data points represent the mean of 3 measurements and error bars represent one standard deviation from the mean.

Once P(NIPAM) was thermocycled, we determined the absolute mass and relative mass of fluid expelled from $\mathrm{P}$ (NIPAM) after heating the sample for 12 minutes at $40{ }^{\circ} \mathrm{C}$. The duration of the heating step (12 minutes) was an arbitrary amount of time selected because we believe it is a reasonable time scale for the operation of microPADs in the real world. The temperature of $40^{\circ} \mathrm{C}$ was chosen because it is achievable using a HotHands hand warmer, which is an inexpensive, commercially available and portable heat source, and it is conceivable that a health professional could use this type of product in the field to perform a paper-based diagnostic assay with P(NIPAM) stored on the device. 
The mass of fluid delivered by the P(NIPAM) sample was strongly dependent on the surface area of the P(NIPAM) exposed to the heat source. For samples of the same initial mass, the sample with the largest diameter-and therefore the largest surface area in contact with the heat source-consistently delivered the most fluid (Figure 3.1.3). We also observed that the amount of fluid delivered eventually reached a plateau as the initial mass of the sample was increased. This result suggests that the amount of fluid delivered by the sample is limited by the area of the sample exposed to the heat source. Fortunately, most microPADs require only small volumes $(5-50 \mu \mathrm{L})$ of fluid to operate, and these volumes can be achieved using the $11.03 \mathrm{~mm}$ and $7.24 \mathrm{~mm}$ diameter P(NIPAM) samples. In Figure 3.1.4, we see that the average mass percent delivered approaches zero as the initial mass of the sample increases indicating again that the surface area is the major determinant of the mass expelled from the hydrogel. Additionally, Figure 3.1.4, is useful because it will allow users to predict the mass of fluid that will be delivered by a given P(NIPAM) sample when heated at $40{ }^{\circ} \mathrm{C}$ for 12 minutes. This general method could be used to make a set of plots for different heating temperatures and time intervals, allowing users to predict the required $\mathrm{P}($ NIAPM) mass and diameter required to deliver a specific volume of fluid under certain conditions. 


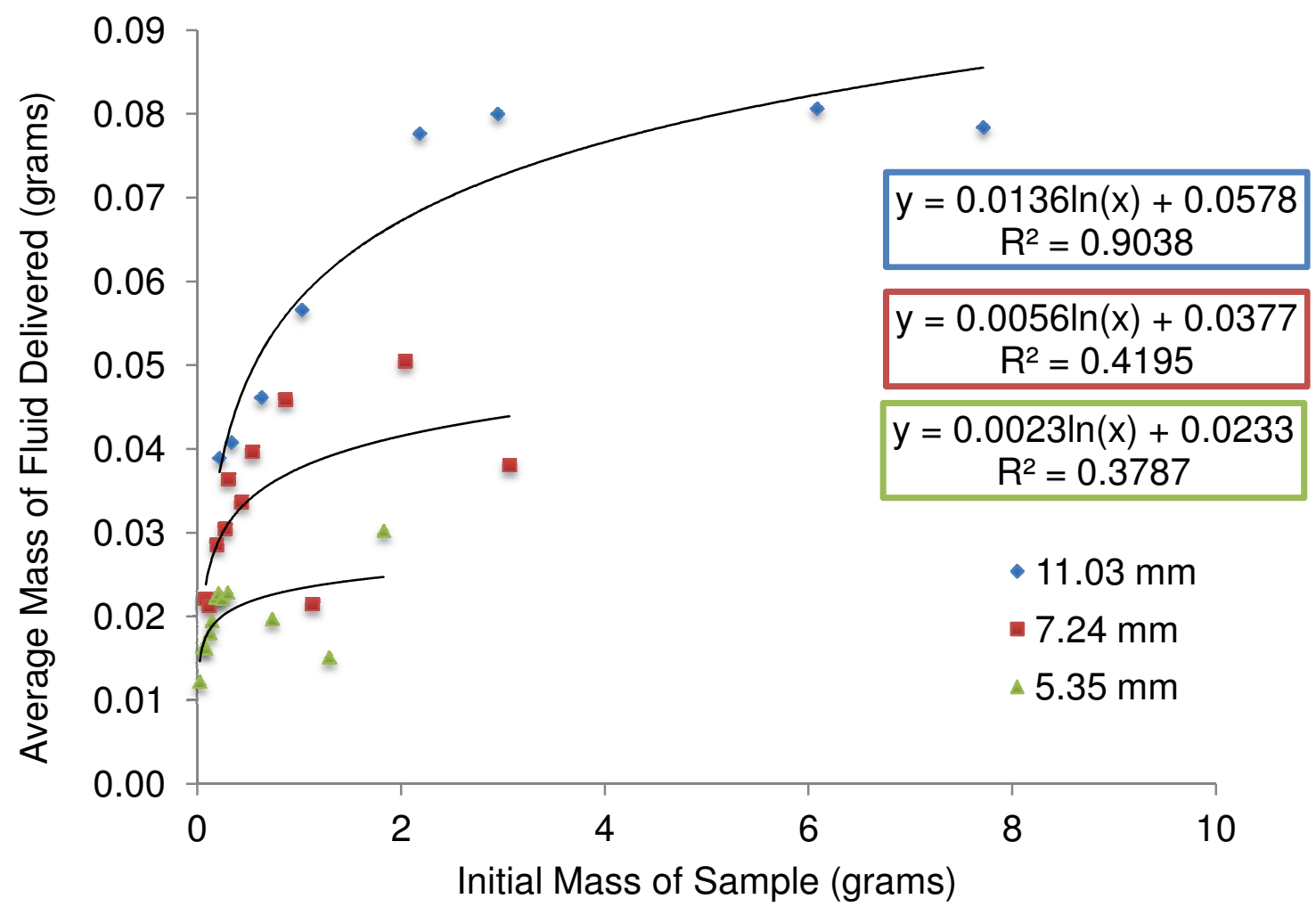

Figure 3.1.3. Plot of the initial mass of a $P(N I A P M)$ sample versus the average mass of fluid expelled. The mass of fluid delivered increases with an increase in the mass of the sample. Diminishing returns are observed. Data points represent the mean of 3 measurements. Data was fit with logarithmic trend lines to aid the eye. 


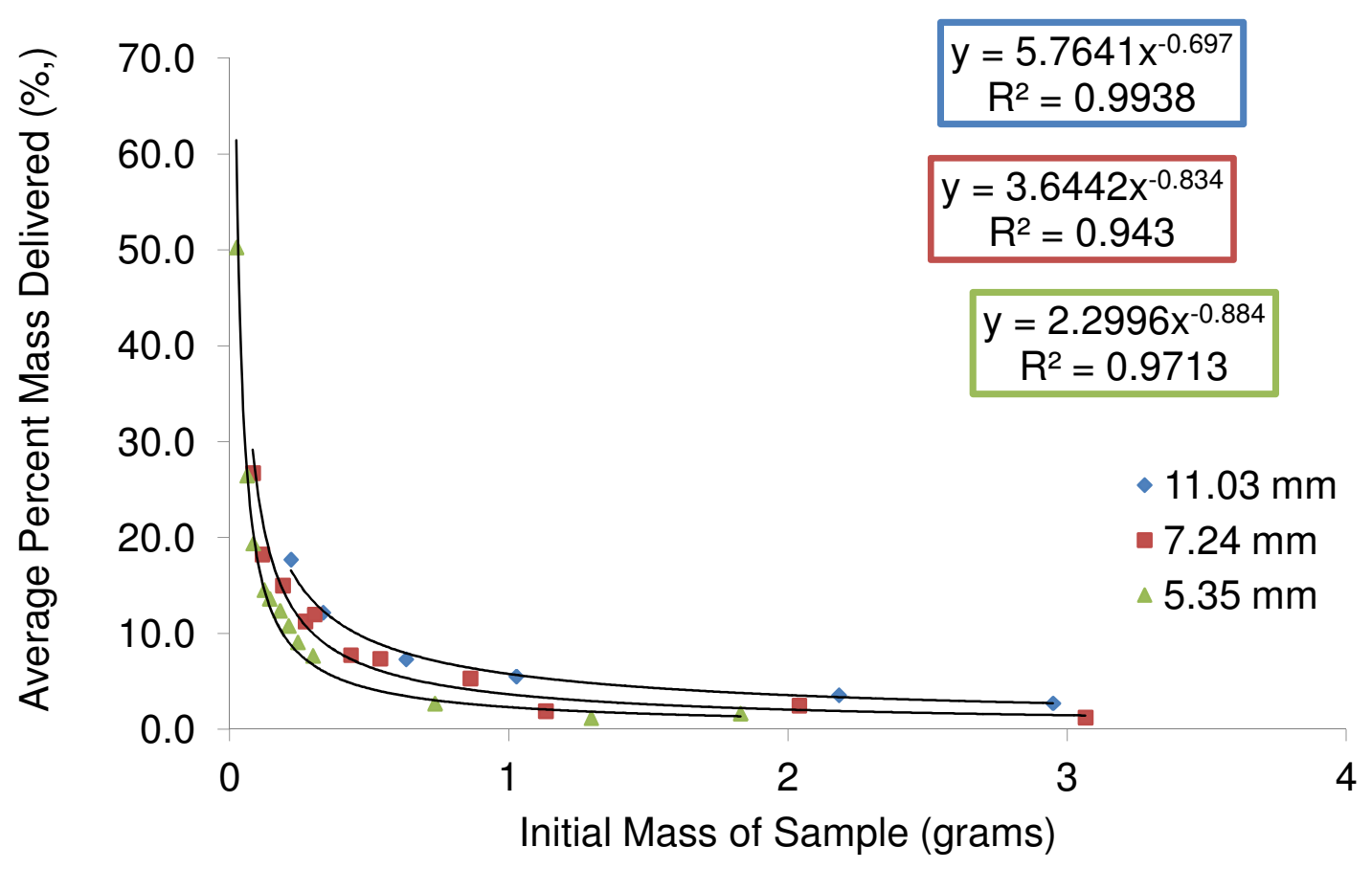

Figure 3.1.4. Plot of the initial mass of P(NIPAM) sample versus the average percent mass delivered. The average percent mass delivered was relative to the initial mass of the sample. The mass of fluid delivered relative to the initial mass of the P(NIPAM) sample precipitously decreases and levels near $0.5 \mathrm{~g}$ for each of the three diameters. Data points represent the mean of 3 measurements and error bars represent one standard deviation from the mean. Data was fit with power function trend lines to aid the eye.

The effect of the surface area and mass of the P(NIPAM) sample was of interest in designing P(NIPAM) samples appropriate for use on microPADs. For completeness of the investigation of P(NIPAM), the effect of time on mass delivered for two sample masses of the three diameters was studied. Figures 
3.1.5, 3.1.6 and 3.1.7 show that mass delivered increases for longer heat exposures times. The results are also consistent with earlier results showing that larger masses expel more fluid. The large deviations and noise seen in Figures 3.1.5, 3.1.6 and 3.1.7 are likely because only one trial was conducted for each sample.

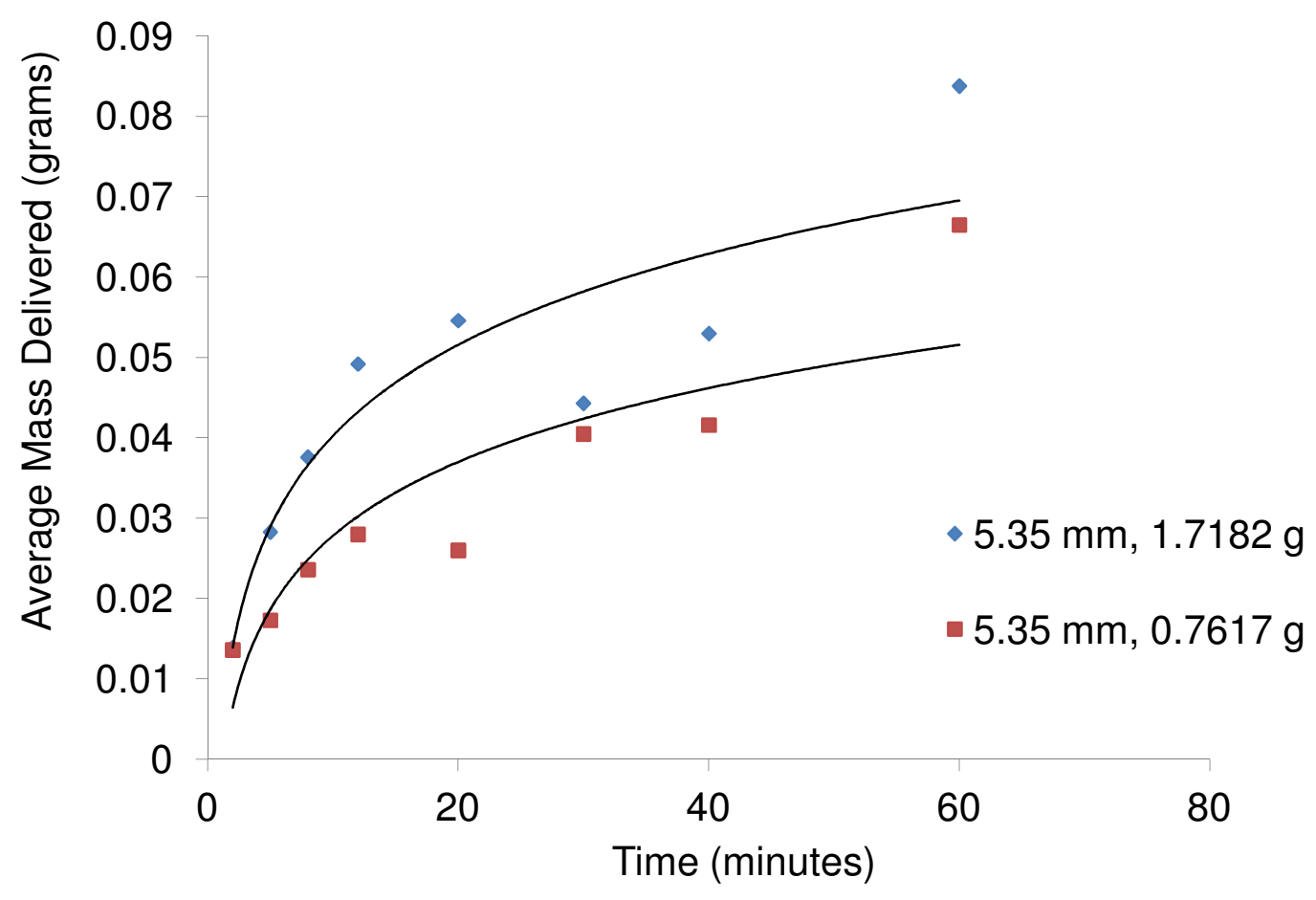

Figure 3.1.5. Plot of heating time versus average mass delivered from $5.35 \mathrm{~mm}$ diameter samples. Each data point represents a single measurement. Data was fit with logarithmic trend lines to aid the eye. 


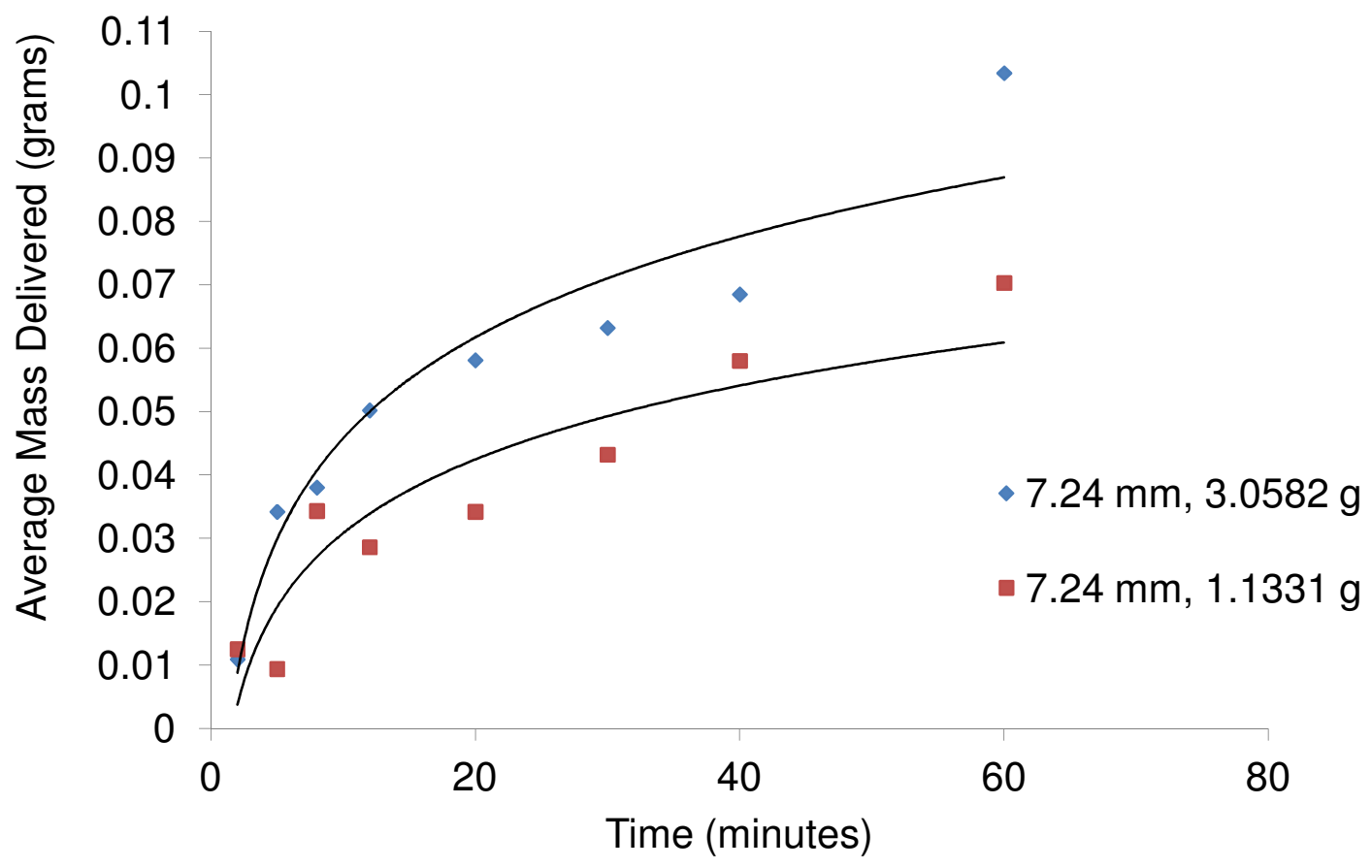

Figure 3.1.6. Plot of heating time versus average mass delivered from $7.24 \mathrm{~mm}$ diameter samples. Each data point represents a single measurement. Data was fit with logarithmic trend lines to aid the eye. 


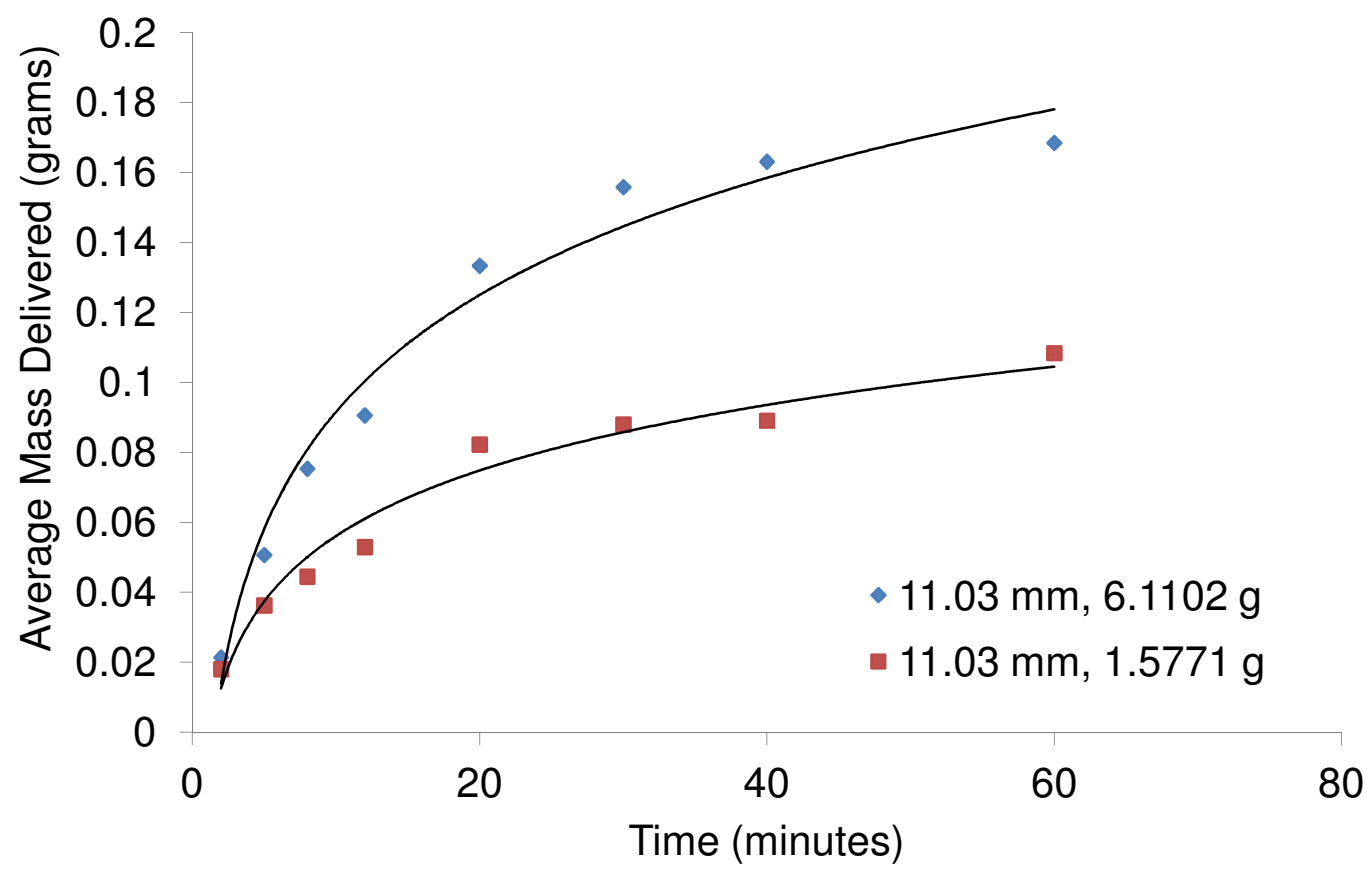

Figure 3.1.7. Plot of heating time versus average mass delivered from $11.03 \mathrm{~mm}$ diameter samples. Each data point represents a single measurement. Data was fit with logarithmic trend lines to aid the eye.

\subsection{Preliminary P(NIPAM) Applications with MicroPADs}

We found that P(NIPAM) can effectively deliver fluids to a microPAD from a traditional circular sample inlet zone (Figure 3.2.1). The wicking characteristics of the system are comparable to those observed for fluid delivered directly to the sample inlet using a micropipette (Figure 3.2.2). ${ }^{40}$ An interesting observation was that the P(NIPAM) samples delivered fluid to the microPAD even at room temperature, which was below the LCST of the polymer. Fluid delivery at room 
temperature is suspected to occur due to the fluid interacting favorably with paper and thus being wicked out of the P(NIPAM) matrix. When the P(NIPAM) was heated to $40{ }^{\circ} \mathrm{C}$, even more fluid was delivered to the device, and the wicking rate increased compared to room temperature conditions. After 30 minutes, the heated P(NIPAM) sample delivered a similar volume of fluid to the channel as compared to the experiment where solution was pipetted directly onto the sample addition zone, but with a different wicking rate profile. Both of these results are interesting because they suggest that $\mathrm{P}(\mathrm{NIPAM})$ and temperature could be used to tune the wicking rate and total mass of fluid delivered to a microPAD, which could be useful for controlling the movement of fluids in a device. This temperature control over wicking rate may allow for fluids to be delivered in a specific sequence from various P(NIPAM) samples on the same device by selectively heating the gels. This could have applications for a P(NIPAM)-powered ELISA. 


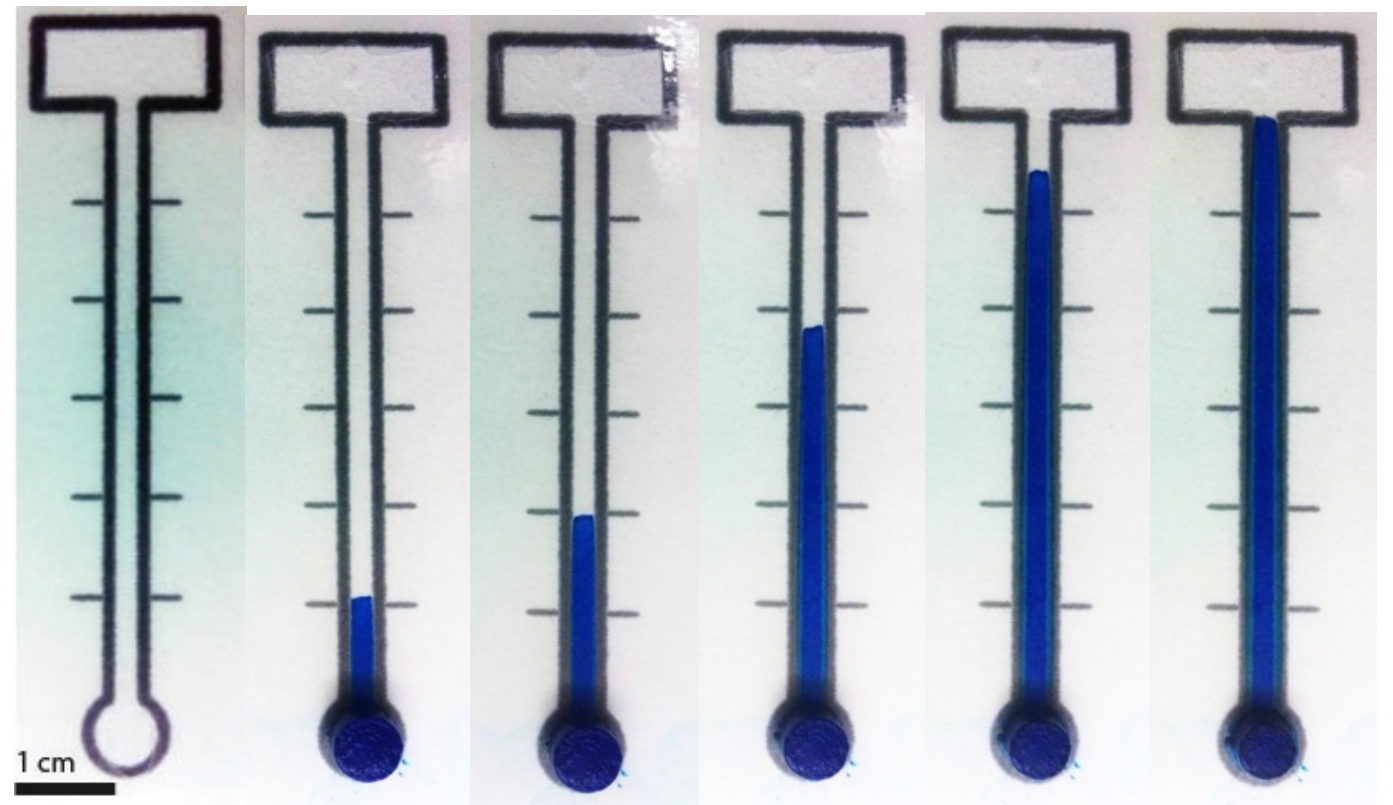

time $=0 \mathrm{~min} \quad 2 \mathrm{mins} \quad 6 \mathrm{mins} \quad 20 \mathrm{mins} \quad 40 \mathrm{mins} \quad 60 \mathrm{mins}$

Figure 3.2.1. A sequence of images documenting the diminishing wicking rate of fluid delivered to a circular sample addition zone of a microPAD by P(NIPAM) at $40{ }^{\circ} \mathrm{C}$. The diminishing wicking rate is typical of fluids in microPADs. 


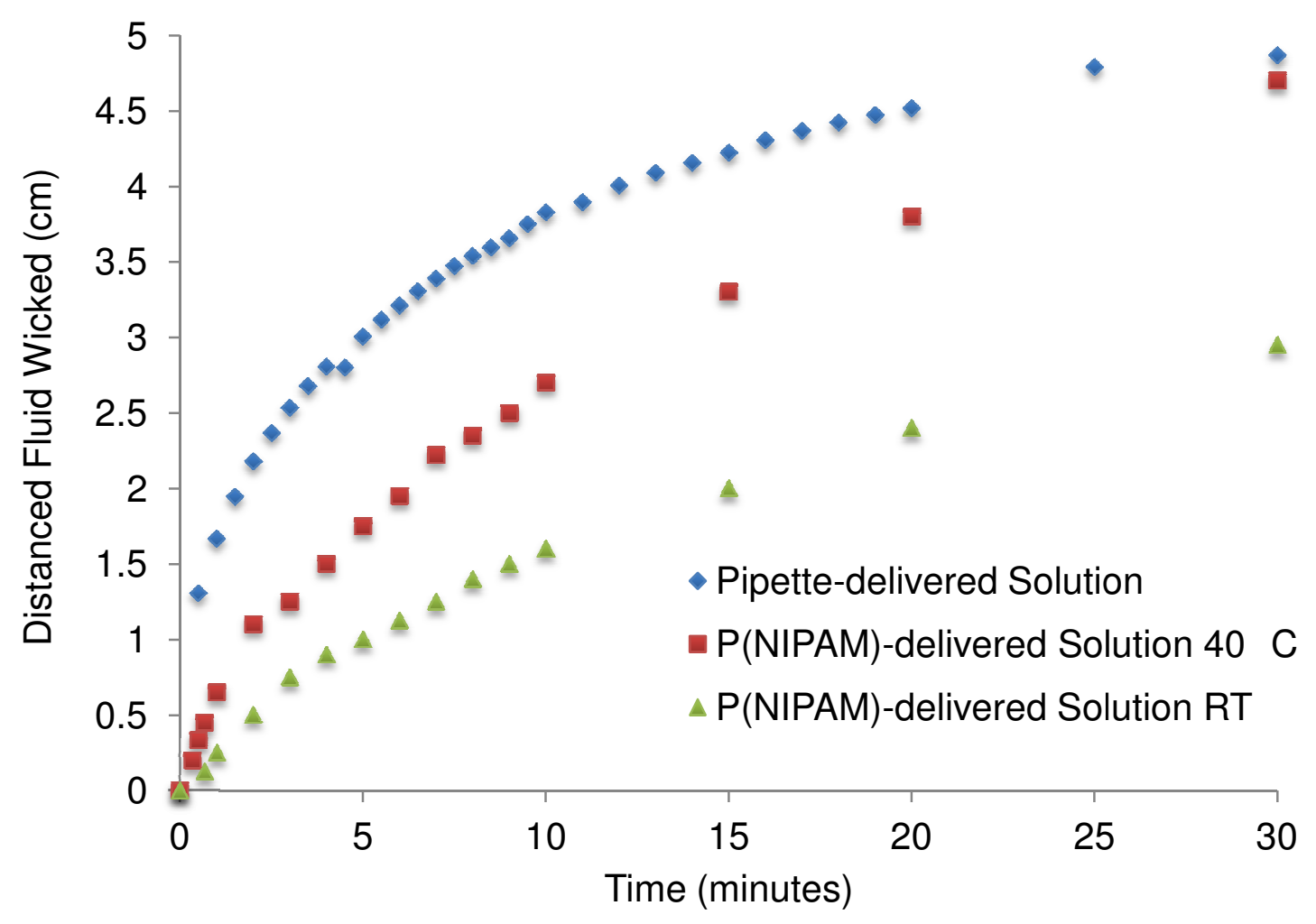

Figure 3.2.2. A plot of time versus distance wicked for fluids delivered to the same device by three different methods. Data points for the pipette-delivered solution $(\checkmark)$ represent the mean of 3 trials. Data points for both P(NIPAM)delivered solutions represent a single measurement. The pipette-delivered solution was borrowed from Camplisson et al. ${ }^{40}$

Once the basic delivery of fluids from P(NIPAM) to microPADs was demonstrated, devices were designed to demonstrate P(NIPAM) could deliver fluids in two classic microfluidic configurations: laminar flow and plug-reagent delivery, Figures 3.2.3 and 3.2.4, respectively. We see these experiments as a 
first step toward demonstrating higher-level functionality of microPADs with P(NIPAM). Laminar flow and sequential addition could serve diagnostic as well as academic purposes. ${ }^{16,41}$ The first device, Figure 3.2.3, demonstrates $\mathrm{P}$ (NIPAM) can deliver fluids simultaneously from neighboring sample zones into a single channel and that the fluids participate in laminar flow. A gradient of both blue and red dye may be seen at 60 minutes. This suggests the simultaneous addition of fluids could be used to produce a gradient of a particular reagent across the width of a channel. If a gradient of an analyte loaded into the hydrogel was produced, it may be possible to create a calibration curve from a single experiment.

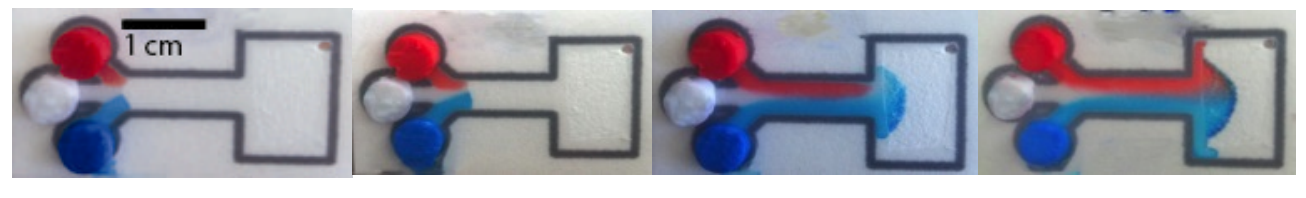

time $=\quad 1 \mathrm{~min} \quad 5 \mathrm{mins} \quad 40 \mathrm{mins} \quad 60 \mathrm{mins}$

Figure 3.2.3. A sequence of images documenting three loaded P(NIPAM) samples delivering fluids simultaneously. These fluids then participate in laminar flow. Dye diffusion can be seen across the channel between the three fluids.

The second device demonstrates P(NIPAM)'s ability to add fluids in a sequence with minimal mixing in the channel (Figure 3.2.4). This sequential addition would be useful for performing a multi-step assay such as an ELISA. An 
antibody would be immobilized on the paper, a sample with biological analyte would be added, followed by the deposition of two P(NIPAM) gels. One gel would be loaded with a secondary enzyme-labeled antibody, and the other with an enzyme substrate that would produce a color change after reacting with the enzyme.

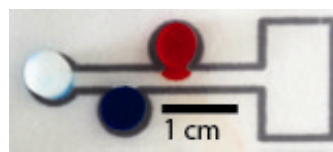

time $=1 \mathrm{~min}$
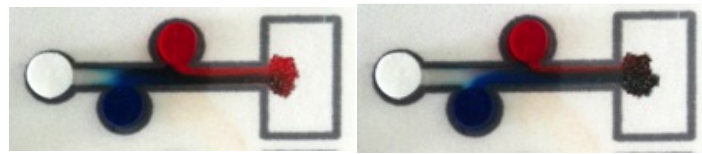

30 mins

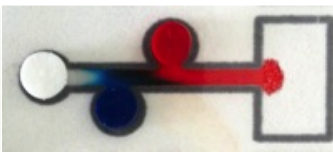

10 mins

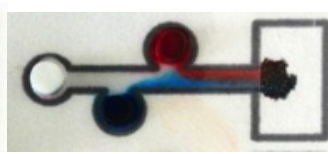

110 mins

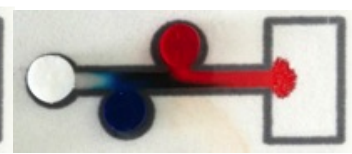

12 mins

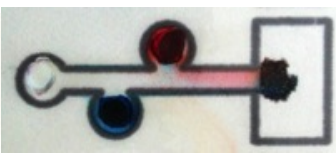

130 mins

Figure 3.2.4. A sequence of images documenting three loaded P(NIPAM) samples delivering fluids simultaneously. These fluids then participate in plugreagent delivery. Dye diffusion is minimal between the solutions.

\subsection{Delivery of Clinically Relevant Molecules using P(NIPAM)}

P(NIPAM) was used successfully to store and then deliver glucose, fluorescein-labeled rabbit anti-sheep IgG, fluorescein-labeled DNA, and HRP to microPADs, which are representative of four major classes of analytes that are 
interesting for diagnostic purposes: small molecules, antibodies, DNA, and enzymes.

\subsubsection{Delivery of Glucose}

Figure 3.3.1.1 shows the results of three trials for seven samples from three conditions of glucose solutions: Condition 1, Condition 2, and Condition 3. Condition 1 is P(NIPAM) loaded with glucose solution. Condition 2 is the excess glucose solution remaining in the vials used to load the P(NIPAM). Condition 3 is the external glucose solutions (section 2.5.2.1). The signals of Condition 1 are of comparable intensity to the signals from both Condition 2 and Condition 3 for the concentrations of glucose that were tested (Figure 3.3.1.2). This result suggests that the same concentrations of glucose were delivered to the test zones via all three conditions. This is important because it demonstrates that (i) P(NIPAM) can deliver glucose to microPADs in a concentration-dependent fashion, (ii) loading glucose into P(NIPAM) has little to no effect on the concentration of glucose in solution, and (iii) delivering glucose to microPADs from P(NIPAM) has little to no effect on the concentration of glucose in solution. The results indicate that glucose is not being retained by or excluded from the P(NIPAM) matrix. This is promising first evidence that small molecules, at least of similar polarity to glucose, could be loaded into P(NIPAM) and delivered to microPADs without affecting their concentration. The results also suggest that P(NIPAM) samples 
loaded with standard solutions could be taken into the field and used to prepare external calibration curves at the point-of-care.

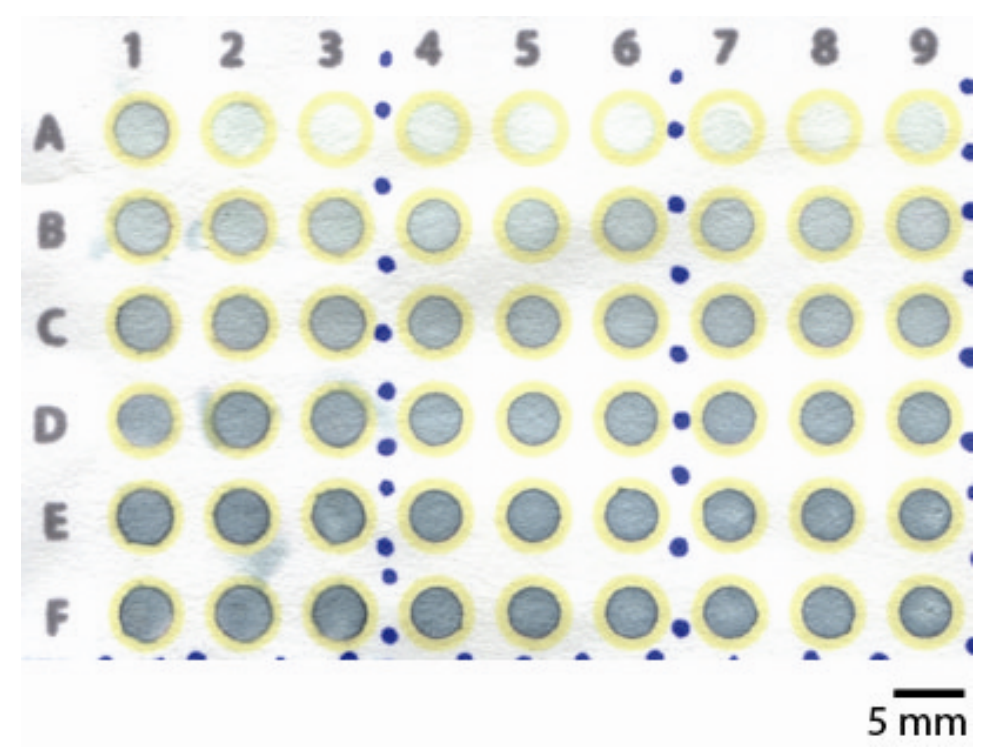

Condition 1 Condition 2 Condition 3

Figure 3.3.1.1. Scanned image of the P(NIPAM) delivered glucose solutions onto a paper-based 96-well plate (section 2.5.2.1). Concentration of glucose delivered increases from row A to F. Condition 1, Condition 2 and Condition 3 was applied to columns $1-3,4-6$, and 7-9, respectively. Reaction zone A1 was contaminated by a $1 \mathrm{mM}$ glucose loaded P(NIPAM) sample. 


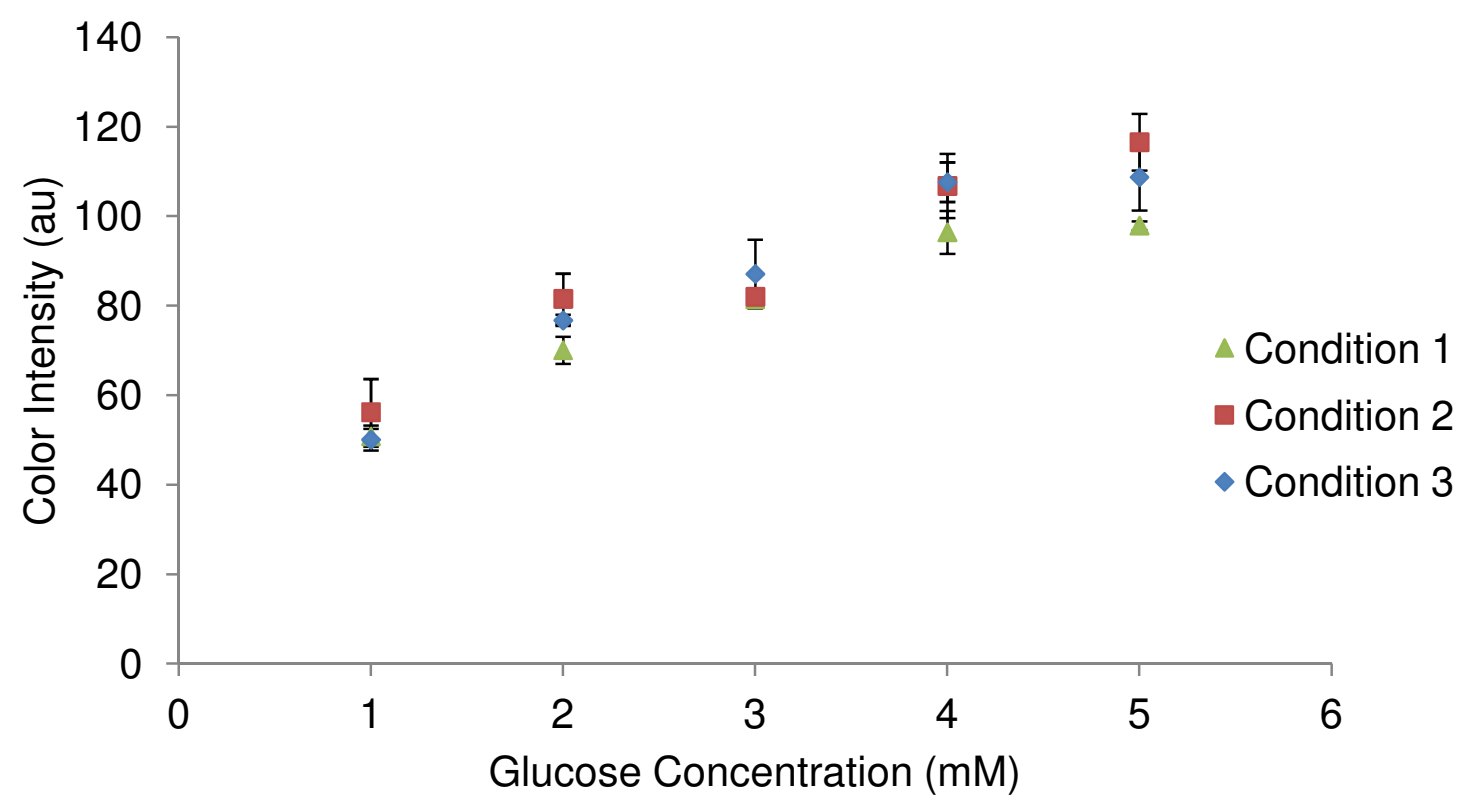

Figure 3.3.1.2 Plot of color intensity versus the glucose concentration for Conditions 1-3. Data points represent the mean of 3 measurements and error bars represent one standard deviation from the mean.

The results from P(NIPAM)-delivered glucose solutions (0mM to $5 \mathrm{mM}$ ) were used to construct an external calibration curve for glucose (Figure 3.3.1.3). Colorimetric signals for glucose assays typically follow a rectangular hyperbolic trend and the glucose solutions used in this experiment were on the high end of the colorimetric signal's rectangular hyperbolic trend. We chose to fit the data with a linear trendline to simplify its use for determining the concentration of unknown samples. This is the reason for the large $y$-intercept value and poor $R^{2}$ value (0.9299). In spite of the poor fit, the calibration curve can still be used to 
estimate the concentrations of glucose in unknown solutions in a clinicallyrelevant range of concentrations $(0 \mathrm{mM}$ to $5 \mathrm{mM}){ }^{2,27}$

The external calibration curve was used to quantify the concentration of two glucose samples delivered by P(NIPAM) and two glucose solutions delivered by pipette (Table 3.3.1.1). The determined concentrations of all four glucose samples were found to be within one standard deviation of their true concentrations. The magnitude of the standard deviations was approximately $10-15 \%$ of the magnitude of the concentrations. The accuracy and precision of these results is comparable to the results obtained typically for microPADs using pipet-delivered solutions. ${ }^{2}$ Ultimately, these results strengthen the proposition that $\mathrm{P}$ (NIPAM) could be used for preparing external calibration curves with microPADs, and also suggest that samples could be collected and stored in the field by loading them into P(NIPAM) gels. 


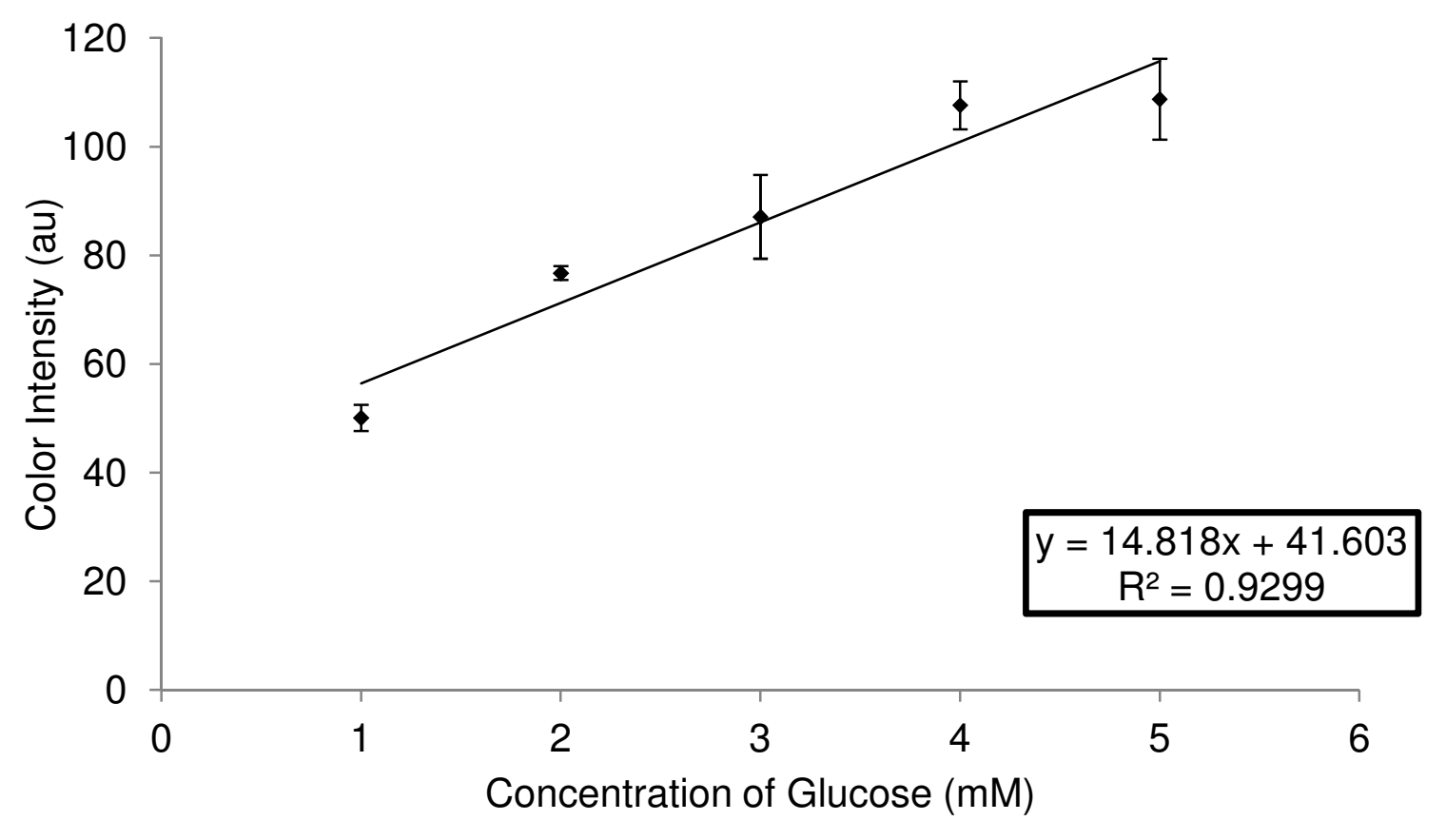

Figure 3.3.1.3. Plot of concentration of glucose versus color intensity for the P(NIPAM)-delivered solutions. The equation of the best-fit line for this plot may be used to determine the concentration of glucose in samples for concentrations between $0 \mathrm{mM}$ and $5 \mathrm{mM}$ glucose. Data points represent the mean of 3 measurements and error bars represent one standard deviation from the mean. 
Table 3.3.1.1. The calculation of the concentration of glucose in samples using the $\mathrm{P}$ (NIPAM) produced glucose calibration curve. Results represent the mean of 3 measurements.

\begin{tabular}{lccc}
\hline $\begin{array}{c}\text { Calibration Curve } \\
\text { (Glucose) }\end{array}$ & $\begin{array}{c}\text { Known } \\
\text { [Glucose] }[\mathrm{mM}]\end{array}$ & $\begin{array}{c}\text { Experimentally determined } \\
\text { [Glucose] }[\mathrm{mM}]\end{array}$ & $\begin{array}{c}\text { Standard Deviation } \\
\text { (x-value) }\end{array}$ \\
\hline P(NIPAM) Delivered & 2.5 & 2.6 & 0.5 \\
External Solution & 2.5 & 2.5 & 0.2 \\
P(NIPAM) Delivered & 3.5 & 3.6 & 0.4 \\
External Solution & 3.5 & 3.4 & 0.5 \\
\hline
\end{tabular}

\subsubsection{Delivery of Fluorescein-labeled IgG}

Figure 3.3.2.1 shows that P(NIPAM) delivers fluorescein tagged antibody, and that the amount delivered is dependent on the concentration of antibody loaded into the P(NIPAM) samples. The plot of the signal for P(NIPAM)-delivered antibody in Figure 3.3.2.1 is linear and is similar to the results obtained for the pipette delivered antibody. The P(NIPAM) delivered antibody has greater deviation due most likely to the poor design of the experiment. The solutions delivered by P(NIPAM) did not completely fill the devices because the test zone and channel were too large for the mass of P(NIPAM) used, and thus a non-ideal method of signal quantification had to be implemented (section 2.5.2.3). We believe the results of this experiment could be improved by using an appropriate mass of $\mathrm{P}($ NIPAM) to deliver the antibody, but, even in their current state, the 
results show potential for using $\mathrm{P}(\mathrm{NIPAM})$ to store and deliver antibodies for the preparation of calibration curves.

The results shown in Table 3.3.2.1 corroborate that the P(NIPAM)delivered antibody calibration curve is unreliable for determining samples of unknown concentrations within the range of the calibration curve. The determined concentration values were inaccurate (outside one standard deviation) when compared to the true concentrations of the solutions. Surprisingly, the results obtained using the pipet-delivered calibration curve also had poor accuracy, although better than the accuracy for the P(NIPAM)-delivered calibration curve. Additional optimization of this experiment is required before definitive conclusions can be made. 


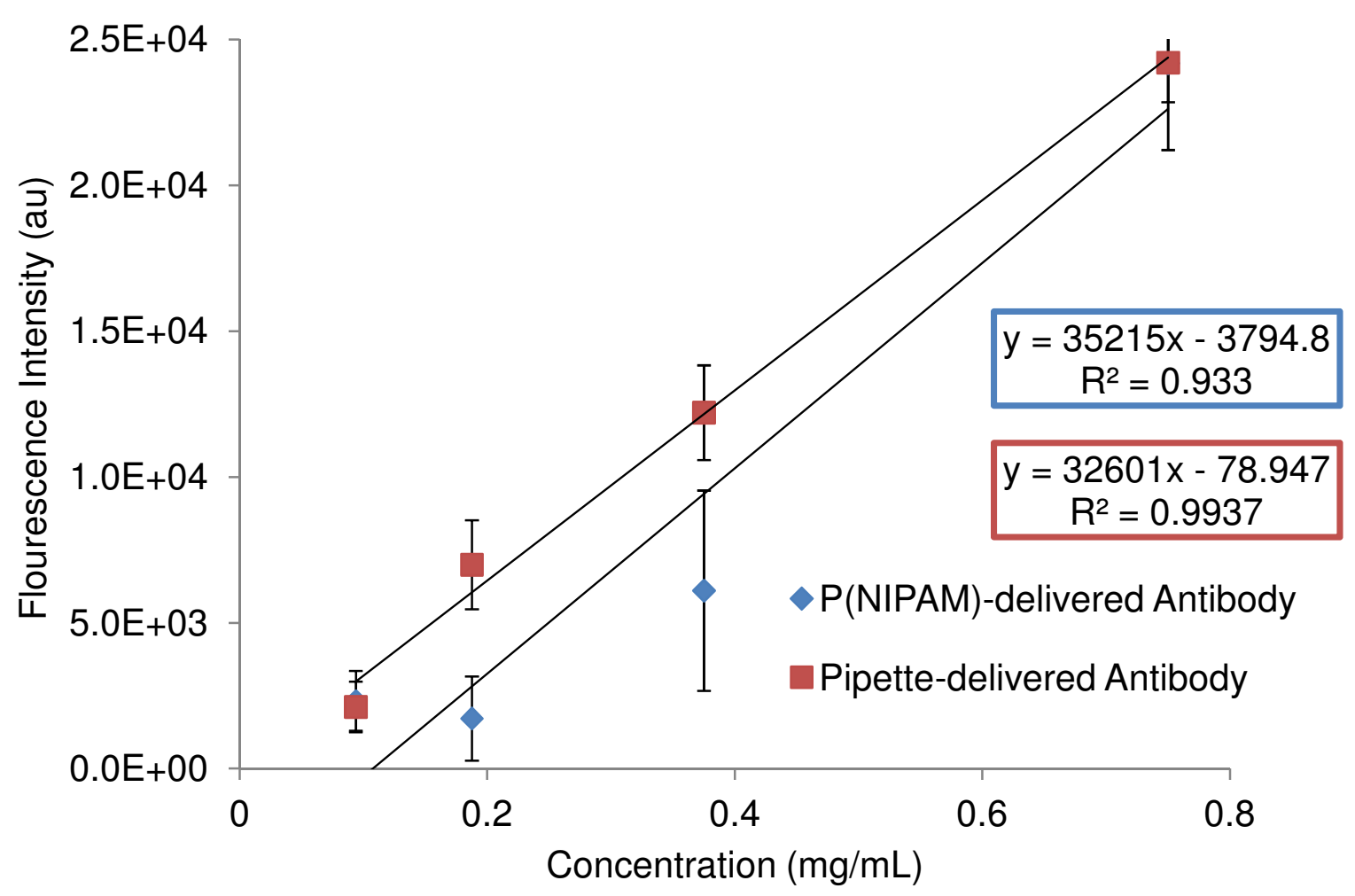

Figure 3.3.2.1. Plot of fluorescence intensity versus concentration for P(NIPAM)delivered fluorescein-labeled antibody $(\diamond)$ and pipette-delivered fluoresceinlabeled antibody (घ). Data points represent the mean of 3 measurements and the error bars represent one standard deviation from the mean. 
Table 3.3.2.1. The determined concentrations of antibody samples based on their fluorescence intensity signal using calibration curves produced using P(NIPAM)-delivered and a pipette-delivered standard antibody solutions. Results represent the mean of 3 measurements.

\begin{tabular}{lccc}
\hline $\begin{array}{c}\text { Kalibration Curve } \\
\text { (Antibody) }\end{array}$ & $\begin{array}{c}\text { Experimentally } \\
\text { [Antibody] } \\
(\mathbf{m g} / \mathbf{m L})\end{array}$ & $\begin{array}{c}\text { determined } \\
\text { [Antibody] } \\
(\mathbf{m g} / \mathbf{m L})\end{array}$ & $\begin{array}{c}\text { Standard Deviation } \\
(\mathbf{m g} / \mathbf{m L})\end{array}$ \\
\hline P(NIPAM)-Delivered & 0.125 & 0.19 & 0.03 \\
Pipette-Delivered & 0.125 & 0.14 & 0.02 \\
P(NIPAM)-Delivered & 0.25 & 0.47 & 0.08 \\
Pipette-Delivered & 0.25 & 0.36 & 0.02 \\
\hline
\end{tabular}

\subsubsection{Delivery of Fluorescein-labeled DNA}

The results obtained for the delivery of DNA using P(NIPAM) were similar to those obtained for the delivery of antibody. Figure 3.3.3.1 demonstrates that P(NIPAM) delivers fluorescein-labeled DNA, and that the amount of DNA delivered is dependent on the concentration of DNA that was loaded into the P(NIPAM) samples. The results for P(NIPAM)-delivered DNA are similar to those obtained for pipette-delivered DNA. The results for the P(NIPAM)-delivered DNA also have a large standard deviation due to the poor design of the experiment. The trend in signal for DNA is large enough to confirm the solution concentration delivered is dependent on the concentration of the loading solution. Regardless of the imperfect device design and non-ideal quantification method, the results 
show potential for P(NIPAM) to produce calibration curves for fluorescein-labeled DNA.

The calibration curves were used to quantify the concentration of DNA in two pipette-delivered samples (Table 3.3.3). These results show slightly better accuracy as compared to the experiments with the antibody, but poor precision, with standard deviations as high as $46 \%$ of the determined concentration. The results suggest that these experiments require further optimization before P(NIPAM) could be used to deliver DNA for quantitative measurements. The results also suggest there may be inherently more determinate and indeterminate error associated with the method used to quantify the results. The results of the experiments with DNA and antibody were quantified using a fluorescence scanner, while all the other results were quantified using a desktop scanner. It would be helpful to conduct a colorimetric assay for the antibody and DNA to see if this reporting system improved the accuracy and precision of the results. 


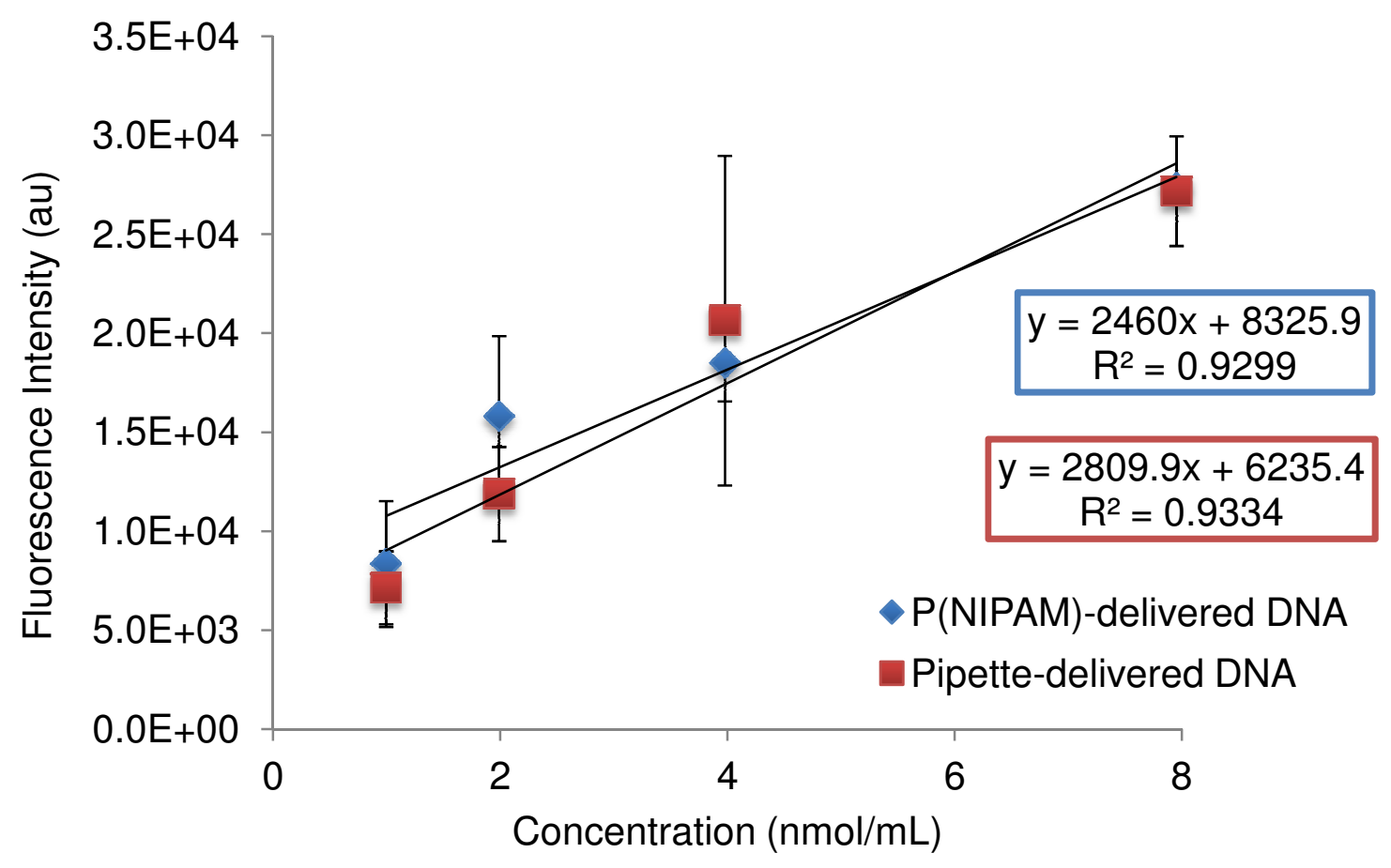

Figure 3.3.3.1. Plot of fluorescence intensity versus concentration for P(NIPAM)delivered fluorescein-labeled DNA $(\diamond)$ and pipette-delivered DNA (匹). Data points represent the mean of 3 measurements and error bars represent one standard deviation from the mean. 
Table 3.3.3.1. Determined concentrations of DNA samples based on their fluorescence intensity signal using the calibration curves produced from P(NIPAM)-delivered and a pipette-delivered DNA. Results represent the mean of 3 measurements.

\begin{tabular}{lccc}
\hline $\begin{array}{c}\text { Calibration Curve } \\
\text { (DNA) }\end{array}$ & $\begin{array}{c}\text { Known } \\
{[\mathrm{DNA}]}\end{array}$ & $\begin{array}{c}\text { Experimentally } \\
\text { determined [DNA] } \\
{[\mathrm{nmol} / \mathrm{mL}]}\end{array}$ & $\begin{array}{c}\text { Standard Deviation } \\
{[\mathrm{nmol} / \mathrm{mL}]}\end{array}$ \\
\hline P(NIPAM)-delivered & 0.9088 & 0.8 & 0.2 \\
Pipette-delivered: & 0.9088 & 1.3 & 0.6 \\
P(NIPAM)-delivered & 3.635 & 2.9 & 0.5 \\
Pipette-delivered: & 3.635 & 3 & 1 \\
\hline
\end{tabular}

\subsubsection{Delivery of HRP}

P(NIPAM) can deliver HRP to paper via passive wicking (Figure 3.3.4.1). The appearance of the blue-green color from oxidized ABTS confirms that the enzyme was delivered to the paper test zone and was still active. Both HRP impregnated $\mathrm{P}(\mathrm{NIPAM})$ samples showed a visually comparable signal to the external HRP solution. Once the ability to deliver HRP was confirmed, the $\mathrm{P}($ NIPAM) samples were reused three more times (section 2.5.2.2). Figure 3.3.4.2 shows that each use of the P(NIPAM) samples produced a signal that was comparable to the last. The signals from the P(NIPAM) samples were also consistently larger than the signals from the External Solution samples, but this is probably because more HRP solution was delivered to the test zone from the 
P(NIPAM). The results shows that P(NIPAM) samples could possibly be recycled for numerous uses without any loss in effective concentration of enzyme delivered. This could be a powerful tool to microPADs. The ability to reuse and even possibly recycle (flush out old reagents and load new reagents) P(NIPAM) would result in little material required, lowering the overall cost of using P(NIPAM) with microPADs. An end-user could potentially work with one set of reagent-loaded P(NIPAM) gels for an extended period of time, reusing them for multiple assays.

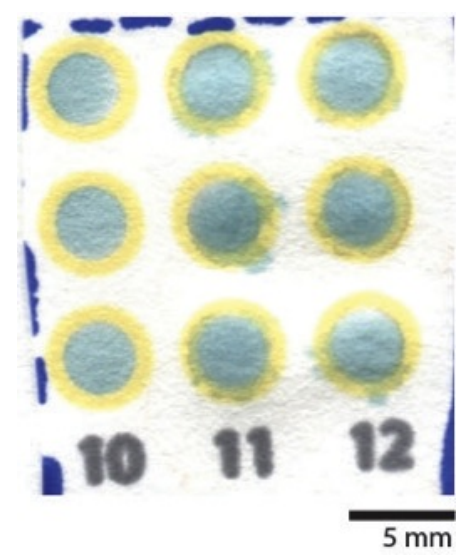

Figure 3.3.4.1. Image of P(NIPAM)-delivered HRP (section 2.5.2.2) in the wells of a paper-based 96 -well plate. Column 10 is the External Solution trials. Column 11 is the Solution Storage trials. Column 12 is the Nonsolution Storage trails. 


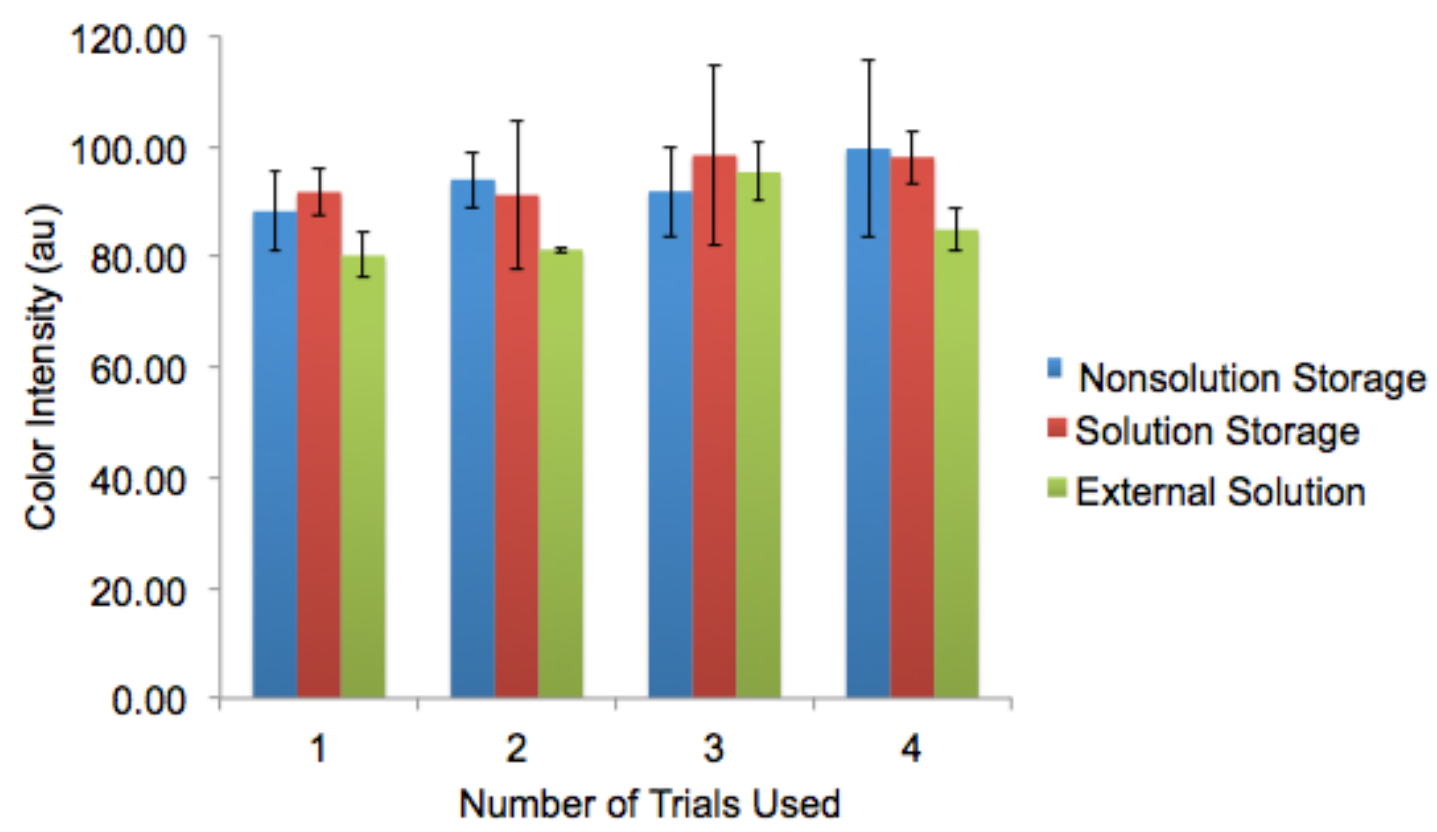

Figure 3.3.4.2. Plot of color intensity versus the number of trials a sample was used. Data points represent the mean of 3 measurements and error bars represent one standard deviation from the mean.

\subsection{Shelf Life of HRP in P(NIPAM)}

Figure 3.4.1 compares the activity of HRP for three different storage conditions as a function of time. The first storage condition was HRP in P(NIPAM) and stored in a dry vial (Nonsolution Storage). The second was HRP in P(NIPAM) and in a vial full of HRP solution (Solution Storage). The third was HRP in solution (External Solution). The activity of HRP for all three conditions was observed to be comparable for each day of a 35-day trial. This indicates $\mathrm{P}($ NIPAM) has no effect on the stability of HRP, and that P(NIPAM) has the 
potential to be used as a storage matrix for sensitive biological reagents. HRP commonly last only a few days at best when stored on paper, even when using a stabilizing agent such as Trehalose., ${ }^{2,27-32}$ P(NIPAM) keeps the enzyme stable for at least 35 days and probably longer considering that the signal for the assay had only decreased slightly from day 0 . Enzymes for assays could be loaded into a hydrogel, transported into the field at room temperature, and then used to conduct an assay at the point-of-care.

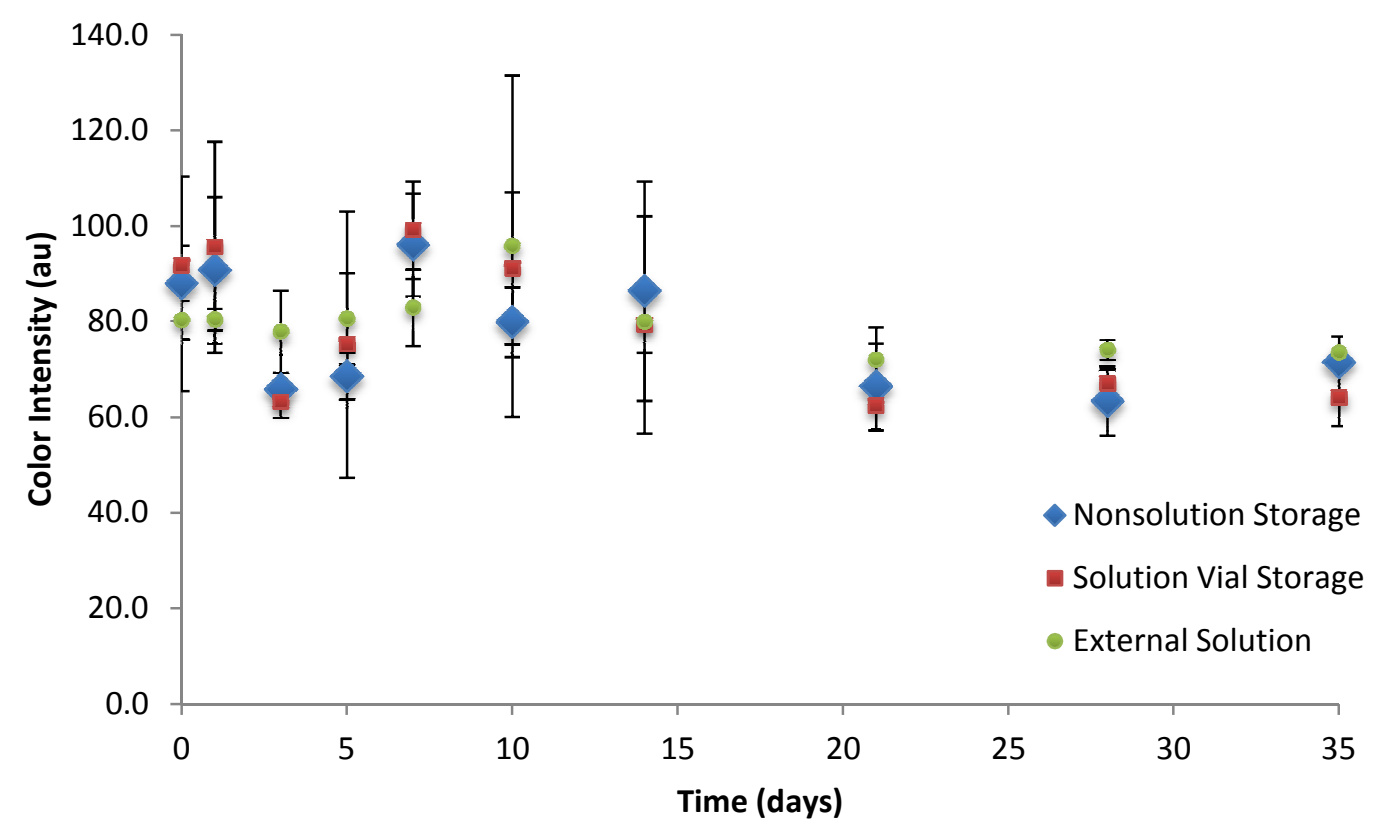

Figure 3.4.1. Plot of color intensity versus time for three conditions (Nonsolution Storage $(\bullet)$, Solution Storage $(\bullet)$, and External Solution $(\bullet))$. Data points represent the mean of 3 measurements and error bars represent one standard deviation from the mean. 


\section{CONCLUSIONS}

A straightforward method for producing consistent samples of P(NIPAM) for delivering fluids to microPADs was designed. The main factor affecting the mass of fluid delivered by a P(NIPAM) sample was the surface area of the sample exposed to the heat source. For a constant surface area, the mass of fluid delivered increases initially with the mass of P(NIPAM) and then plateaus. Additionally, the heating time of the sample was a significant factor in determining the mass of fluid delivered. It should be possible to use the relationships between surface area, initial mass, heating time and the amount of fluid delivered by $\mathrm{P}($ NIPAM) to engineer an appropriate $\mathrm{P}(\mathrm{NIPAM})$ sample for delivery of a specific volume of fluid. It may also be possible to tune the rate at which fluid is delivered from P(NIPAM) samples to microPADs by adjusting the temperature at which the gels are heated., which could be ideal for adding reagents in a specific sequence required for performing an ELISA.

P(NIPAM) showed great potential as a universal material for storing and delivering clinically relevant reagents such as glucose, enzymes, antibodies, and DNA to microPADs. P(NIPAM) was able to deliver glucose solutions to produce an external calibration curve and quantify samples of unknown concentrations within the range of $0 \mathrm{mM}$ and $5 \mathrm{mM}$. A comparable HRP concentration was delivered four times consecutively from the same P(NIPAM) sample, and HRPloaded P(NIPAM) was found to effectively stabilize HRP for 35 days. 
P(NIPAM) has been shown to be a promising fluid delivery system for use with microPADs. This fluid delivery system could enable the development of microPADs capable of relatively complex fluidic operations with minimal input from the user. P(NIPAM) could also be used to produce external calibration curves on the spot while performing diagnostics at the point-of-care. Furthermore, the synthesis and processing of P(NIPAM) is simple, inexpensive, and scalable making it a plausible candidate for use in low-cost diagnostics for the developing world. 


\section{REFERENCES}

1) Initiative, Joint Learning. Human Resources for Health Overcoming the Crisis. N. p.

2) Martinez, Andres W., Scott T. Phillips, George M. Whitesides, et al. "Diagnostics for the Developing World: Microfluidic Paper-Based Analytical Devices." Analytical Chemistry 82 (2010): 3-10.

3) Yager, Paul et al. "Microfluidic Diagnostic Technologies for Global Public Health." Nature 442 (2006): 412-418.

4) Martinez, Andres W., Scott T. Phillips, Manish J. Butte, et al. "Patterned Paper as a Platform for Inexpensive, Low-Volume, Portable Bioassays." Angewandte Chemie - International Edition 46 (2007): 1318-1320.

5) Ellerbee, Audrey K et al. "Quantifying Colorimetric Assays in Paper-Based Microfluidic Devices by Measuring the Transmission of Light through Paper." Analytical chemistry 81.20 (2009): 8447-52.

6) Chin, Curtis D, Vincent Linder, and Samuel K Sia. "Lab-on-a-Chip Devices for Global Health: Past Studies and Future Opportunities." Lab on a chip 7 (2007): 41-57.

7) Chaplan, Cory a., Haydn T. Mitchell, and Andres W. Martinez. "Paper-Based Standard Addition Assays." Analytical Methods 6.5 (2014): 1296. Web. 23 May 2014. 
8) Zhao, Weian, and Albert van der Berg. "Lab on Paper." Lab on a chip 8 (2008): 1988-1991.

9) Yetisen, Ali Kemal, Muhammad Safwan Akram, and Christopher R Lowe. "Paper-Based Microfluidic Point-of-Care Diagnostic Devices." Lab on a chip 13.12 (2013): 2210-51. Web. 24 May 2014.

10) Hsu, Chao-Kai et al. "Paper-Based ELISA for the Detection of Autoimmune Antibodies in Body Fluid-the Case of Bullous Pemphigoid." Analytical chemistry 86.9 (2014): 4605-10.

11) Li, Xu, David R. Ballerini, and Wei Shen. "A Perspective on Paper-Based Microfluidics: Current Status and Future Trends." Biomicrofluidics 2012.

12) Lu, Rao et al. "Rapid Prototyping of Paper-Based Microfluidics with Wax for Low-Cost, Portable Bioassay." Electrophoresis 30 (2009): 1497-1500.

13) Carrilho, Emanuel, Andres W Martinez, and George M Whitesides. "Understanding Wax Printing: A Simple Micropatterning Process for PaperBased Microfluidics." Analytical chemistry 81.16 (2009): 7091-5.

14) Carrilho, Emanuel et al. "Paper Microzone Plates." Analytical Chemistry 81 (2009): 5990-5998.

15) Martinez, Andres W, Scott T Phillips, and George M Whitesides. "ThreeDimensional Microfluidic Devices Fabricated in Layered Paper and Tape." 
Proceedings of the National Academy of Sciences of the United States of America 105.50 (2008): 19606-11.

16) Squires, Todd M., and Stephen R. Quake. "Microfluidics: Fluid Physics at the Nanoliter Scale." Reviews of Modern Physics 77 (2005): 977-1026.

17) Whitesides, George M. "The Origins and the Future of Microfluidics." Nature 442 (2006): 368-373.

18) Whitesides, George M. "A Glimpse into the Future of Diagnostics." Clinical chemistry 59.4 (2013): 589-91. Web. 26 May 2014.

19) Weibel, Douglas B et al. "Torque-Actuated Valves for Microfluidics Place $\square$; on Curing, It Bonds to the Surrounding Layer of of the Threads of the Screws . The Valves Were Separated." 77.15 (2005): 4726-4733. Print.

20) Brody, J P et al. "Biotechnology at Low Reynolds Numbers." Biophysical journal 71.6 (1996): 3430-41. Web. 30 May 2014.

21) Kamholz, A E et al. "Quantitative Analysis of Molecular Interaction in a Microfluidic Channel: The T-Sensor." Analytical chemistry 71.23 (1999): $5340-5347$.

22) Jeon, Noo Li et al. "Generation of Solution and Surface Gradients Using Microfluidic Systems." 15 (2000): 8311-8316. Print. 
23) Jackson, Abby J., Matthew R. Sobansky, and David S. Hage. Antibodies Applications and New Developments. Ed. Eline P. Meulenberg. Bentham Science Publisher, 2012.

24) Institutes, National, and Health Updated October. "Point-of-Care Diagnostic Testing." October (2010): 1-2. Print.

25) Cheng, Chao-Min et al. "Paper-Based ELISA." Angewandte Chemie 122.28 (2010): 4881-4884. Web. 2 June 2014.

26) Murdock, Richard C et al. "Optimization of a Paper-Based ELISA for a Human Performance Biomarker." Analytical chemistry 85.23 (2013): 1163442.

27) Martinez, Andres W., Scott T. Phillips, Emanuel Carrilho, et al. "Simple Telemedicine for Developing Regions: Camera Phones and Paper-Based Microfluidic Devices for Real-Time, off-Site Diagnosis." Analytical Chemistry 80 (2008): 3699-3707.

28) Kaushik, Jai K, and Rajiv Bhat. "Why Is Trehalose an Exceptional Protein Stabilizer? An Analysis of the Thermal Stability of Proteins in the Presence of the Compatible Osmolyte Trehalose." The Journal of biological chemistry 278.29 (2003): 26458-65. Web. 29 May 2014. 
29) Saladino, G. et al. "A Simple Mechanism Underlying the Effect of Protecting Osmolytes on Protein Folding." Journal of Chemical Theory and Computation 7.11 (2011): 3846-3852.

30) Santoro, M M et al. "Increased Thermal Stability of Proteins in the Presence of Naturally Occurring Osmolytes." Biochemistry 31.23 (1992): 5278-83.

31) Veitch, Nigel C. "Horseradish Peroxidase: A Modern View of a Classic Enzyme." Phytochemistry 2004: 249-259. Print.

32) Bruździak, Piotr, Aneta Panuszko, and Janusz Stangret. "Influence of Osmolytes on Protein and Water Structure: A Step to Understanding the Mechanism of Protein Stabilization." The journal of physical chemistry. B 117.39 (2013): 11502-8.

33) Odian, George G. Principles of Polymerization. Hoboken, N. J.: Wiley, 2004. Print.

34) No part of this publication may be reproduced, stored in a retrieval system, or transmitted in any form or by any means, electronic, mechanical, photocopying, recording, scanning, or otherwise, except as permitted under Section 107 or 108 of the 1976 United States Copyright Act, without either the prior written permission of the Publisher, or authorization through payment of the appropriate per-copy fee to the Copyright Clearance Center, Inc., 222 Rosewood Drive, Danvers, MA 01923, 978-750-8400, fax 978-750-4470, or on the web at www.copyright.com. Requests to the Publisher for permission 
should be addressed to the Permissions Department, John Wiley \& Sons, Inc., 111 River Street, Hoboken, NJ 07030, (201) 748-6011, fax (201) 7486008, e-mail: permreq@wiley.com.

35) Meng, Harper, and Jinlian Hu. "Journal of Intelligent Material Systems and Structures A Brief Review of Stimulus-Active Polymers Responsive to." (2010): n. pag.

36) Ono, Yousuke, and Toshiyuki Shikata. "Hydration and Dynamic Behavior of poly(N-Isopropylacrylamide)s in Aqueous Solution: A Sharp Phase Transition at the Lower Critical Solution Temperature." Journal of the American Chemical Society 128.31 (2006): 10030-1.

37) Zhang, Yanjie et al. "Specific lon Effects on the Water Solubility of Macromolecules: PNIPAM and the Hofmeister Series." Journal of the American Chemical Society 127.41 (2005): 14505-10.

38) Tucker, Ashley K., and Mark J. Stevens. "Study of the Polymer Length Dependence of the Single Chain Transition Temperature in Syndiotactic Poly( N -Isopropylacrylamide) Oligomers in Water." Macromolecules 45.16 (2012): $6697-6703$.

39) Image J Microarray Plugin Authors: Bob Dougherty and Wayne Rasband.

40) Camplisson, C. K., Schilling, K. M., Stone, H., Martinez, A. W., manuscript in preparation. 
41) Kamholz, A E et al. "Quantitative Analysis of Molecular Interaction in a Microfluidic Channel: The T-Sensor." Analytical chemistry 71.23 (1999): $5340-5347$ 